\title{
Properties of long quantum walks in one and two dimensions
}

\author{
Luo Hao, ${ }^{1}$ and Peng $\mathrm{Xue}^{2,3}$ \\ ${ }^{1}$ Department of Physics, Renmin University of China, Beijing 100872, China \\ ${ }^{2}$ Department of Physics, Southeast University, Nanjing 211189, China \\ ${ }^{3}$ State Key Laboratory of Precision Spectroscopy, \\ East China Normal University, Shanghai 200062, China
}

(Dated: October 2, 2018)

\begin{abstract}
The quantum walk (QW) is the term given to a family of algorithms governing the evolution of a discrete quantum system and as such has a founding role in the study of quantum computation. We contribute to the investigation of QW phenomena by performing a detailed numerical study of discrete-time quantum walks. In one dimension (1D), we compute the structure of the probability distribution, which is not a smooth curve but shows oscillatory features on all length scales. By analyzing walks up to $N=1000000$ steps, we discuss the scaling characteristics and limiting forms of the QW in both real and Fourier space. In 2D, with a view to ready experimental realization, we consider two types of QW, one based on a four-faced coin and the other on sequential flipping of a single two-faced coin. Both QWs may be generated using two two-faced coins, which in the first case are completely unentangled and in the second are maximally entangled. We draw on our 1D results to characterize the properties of both walks, demonstrating maximal speed-up and emerging semi-classical behavior in the maximally entangled QW.
\end{abstract}

PACS numbers: 03.67.Ac, 05.40.Fb

\section{INTRODUCTION}

Quantum walk $(\mathrm{QW})$ is an extension of the classical random walk, in which the walker is controlled by a quantum mechanical variable [1, 2]. The quantum interference from different walk paths brings about QW novel and interesting features. An motivation for work on QW is developing new quantum algorithms [3 [5], aiming at an exponential speed-up compared to classical algorithms for a certain classes of problem [6 -8] .

On the physical side, QW is a valuable model for studying evolution process from simple quantum protocol: discrete-time QW [9] and continuous-time QW in one dimension (1D) 10]. Deep studies involve multiple coins [11], multiple walkers [12], multiple dimensions [13], and multiple coins with different degrees of entanglement [14 16]. A recent resurgence of interest in the QW has come about to a significant extent because experimental capability has caught up with many of these theoretical proposals, particularly to create walks with complex coin protocols and in two phase-space dimensions [17 26]. However, all of these QWs implementation are restricted to rather small numbers of steps, and some have inherent limits to the size of their phase space.

Despite the large volume of work performed on the QW, we have not been able to find any that address the properties of the system at long evolution times, meaning at large values of the step number $N$. As the challenges in quantum computing move toward the large scale, it would appear that there is a need to understand the behavior of quantum algorithms in the large- $N$ regime. The aim of this paper is to analyze QWs at large $N$ to establish their properties, both universal and specific, and thus effectively to gauge the flow and concentration of information in one particular set of algorithms. We will consider in detail the 1D QW, where we have performed calculations up to $N=1000000$ steps, to establish the scaling characteristics, spatial information content, universality, and limiting form of the probability distribution. We will then turn to different types of possible QW in 2D, where we use our 1D knowledge both directly and to benchmark the additional forms of behavior that emerge, particularly entanglement, accelerated diffusion, and semi-classical limiting distributions.

In this paper we perform a detailed analysis of the discrete-time 1D QW and two fundamental 2D QWs. By calculating probabilities for large numbers $N$ of steps, we investigate the destructive interference, the scaling properties, the frequency content, and the combination of QWs. The structure of the article is as follows. In Sec. II we review the classical random walk and a simple, symmetrical model for understanding the discrete-time QW in 1D, including its analytical solution. Section III presents our numerical results for 1D QWs up to large $N$ and their analysis in both real and Fourier space. In Sec. IV we discuss two typical 2D QWs as a theoretical basis of Sec. V. Borrowing from the understanding developed in $1 \mathrm{D}$, in Sec. V we provide the complete numerical characterization of these two 2D QWs. Section VI provides a brief summary.

\section{ONE-DIMENSIONAL RANDOM WALKS}

\section{A. Classical Random Walk}

A walker standing at the origin of a line flips an unbiased coin and steps to the right if a head comes up or to the left if the result is a tail. After many flips, and taking a fixed number of steps based the coin state, the 


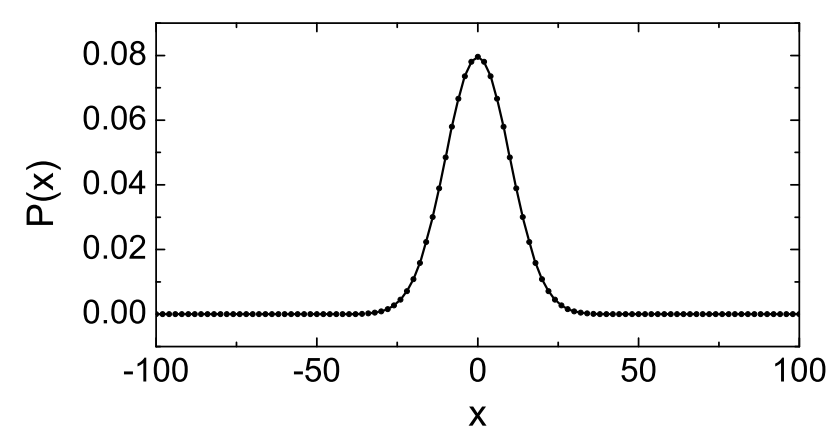

FIG. 1. Probability distribution for a $1 \mathrm{D}$ classical random walk with $N=100$.

location of the walker is an unknown, random position, but the probability distribution of this position is a definite statistical quantity. After $N$ steps, the probability of the walker being at position $x$ is

$$
P_{N}(x)=\frac{1}{2^{N}} C_{N}^{\frac{N-x}{2}}=\left(\frac{1}{2}\right)^{N} \frac{N !}{\left(\frac{N+x}{2}\right)\left(\frac{N-x}{2}\right) !},
$$

i.e. this process, the discrete-time 1D classical random walk, follows a binomial distribution. The walker's position $x$ may only be an even (odd) integer when $N$ is even (odd). In the continuum limit, which is approached for sufficiently large $N$, the distribution is Gaussian (Fig. 1). Its standard deviation, which represents a mean propagation distance in a sample of many walkers, is $\sigma=\sqrt{N}$.

\section{B. Quantum Walks}

A QW refers to a random walk effected using a quantum device, and as such requiring a quantum mechanical description.Following the classical formulation of a coin and a walker, the QW differs fundamentally from its classical counterpart in that coin and walker are "entangled" in the same quantum "particle". The essential consequence of this entanglement is that the propagation of the quantum walker depends not on its probability (Sec. IIA) but on its amplitude. This amplitude is subject to quantum mechanical interference, which can be constructive or destructive, leading to completely unconventional forms of probability distribution (Fig. 2).

The numerical results in Sec $\amalg$ based on following calculation. While there are many ways of describing such a walk (Ref. [1] for a review), here we introduce only the case of a discrete time symmetric QW on an open line. With a view to later application, we denote the two internal "coin" states of the walker, or particle, as $\uparrow$ and $\downarrow$. In a classical walk the coin states are completely separate ( $\uparrow$ or $\downarrow$ with probabilities 0 or 1 ), whereas a quantum coin can occupy any superposition state $a|\uparrow\rangle+b|\downarrow\rangle$. Thus

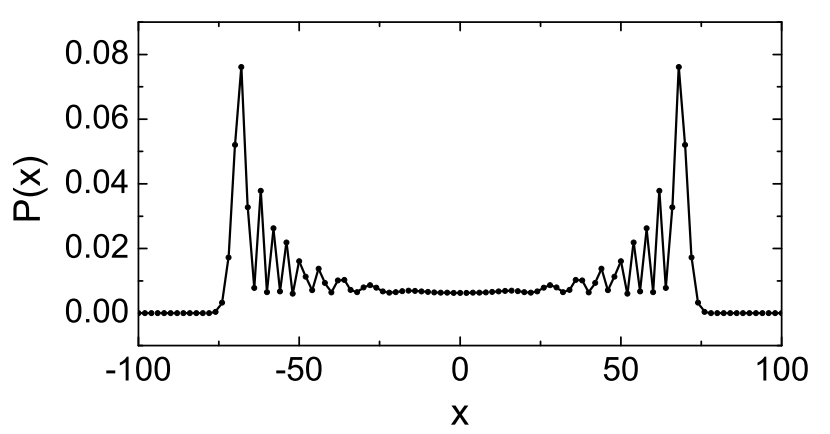

FIG. 2. Probability distribution for a 1D quantum walk with $N=100$.

the quantum system is described by the wave function

$$
\left|\psi_{N}\right\rangle=\sum_{i=-N}^{N}\left(a_{i}|\downarrow\rangle+b_{i}|\uparrow\rangle\right)|i\rangle,
$$

where $N$ is number of steps in the walk, $i$ is the position index, and $|i\rangle$ the corresponding state.

The QW is described by the evolution of this wave function under the quantum operation for successive steps. The probability distribution for finding the walker at position $i$ (state $|i\rangle$ ) is found from the trace over the coin states to be $P_{i}=a_{i}^{2}+b_{i}^{2}$, with $\sum_{i} P_{i}=1$.

The evolution has two substeps: (1) A rotation in the coin space, represented by a unitary operator $U$, which is often taken as the Hadamard transformation

$$
U_{H}=\frac{1}{\sqrt{2}}\left(\begin{array}{cc}
1 & 1 \\
1 & -1
\end{array}\right)
$$

(2) A coin-dependent translation of the walker, described by the shift operator

$$
S=|\uparrow\rangle\left\langle\uparrow\left|\otimes \sum_{i}\right| i+1\right\rangle\langle i|+| \downarrow\rangle\left\langle\downarrow\left|\otimes \sum_{i}\right| i-1\right\rangle\langle i|,
$$

The coefficients $a_{0}$ and $b_{0}$ of the initial coin state are arbitrary, which has a profound influence on the symmetry of the probability distribution of the QW [1]. To obtain a distribution symmetric under the application of the Hadamard operator, the initial coin state is written as

$$
\left|\psi_{0}\right\rangle=\frac{1}{\sqrt{2}}(|\uparrow\rangle+i|\downarrow\rangle) \otimes|0\rangle,
$$

A full step of the QW is

$$
\left|\psi_{1}\right\rangle=S U_{H}\left|\psi_{0}\right\rangle=\frac{1+i}{2}|\uparrow\rangle|1\rangle+\frac{1-i}{2}|\downarrow\rangle|-1\rangle
$$

The probability distribution for a $\mathrm{QW}$ of $N=100$ steps is shown in Fig. 2 It is manifestly completely different from the classical random walk (Fig. 1), with clear maxima at values of $i$ close to $\pm 0.7 N$ (revealed at larger $N$ to be $\pm N / \sqrt{2}$ ). The probability for $i=0$ is close 
to 0 , indicating that the origin of this counter-intuitive behavior is an almost complete destructive interference among the paths of the quantum walker returning to the origin. The standard deviation of the probability distribution in this QW is $\sigma \propto N$, indicating a linear spreading rate, which is one of the most important attributes of this evolution algorithm [Eq. (6)]. We present a quantitative analysis of the properties of the QRW in Sec. III. The QW evolution process acts to propagate the quantum mechanical amplitudes, preserving the complete information content of the internal states.

There are another two kinds of analytical descriptions do benefit to understand the evolution of QW. The difference between the classical and quantum walks can be understood from the non-commuting nature of the (matrix) quantum operators and its consequences for the interference of different walker paths. The Hadamard operator can be decomposed [27] as $U_{H}=P+Q$ with

$$
P=\frac{1}{\sqrt{2}}\left(\begin{array}{ll}
1 & 1 \\
0 & 0
\end{array}\right) \text { and } Q=\frac{1}{\sqrt{2}}\left(\begin{array}{cc}
0 & 0 \\
1 & -1
\end{array}\right) .
$$

It is easy to see that $P$ determines motion of the walker to the right and $Q$ to the left. Evolution under the QW for $N$ steps is represented as $U_{H}^{N}=(P+Q)^{N}$. In the classical random walk one has $1^{N}=(p+q)^{N}$, where $p=q=\frac{1}{2}$ are real numbers representing probabilities.

One may conclude that the non-commutativity of the quantum operators determining different paths to the same walker position, encodes the interference of amplitudes leading to the entirely unconventional quantum phenomena reflected in Figs. 1 and 2, We expand this method to study a novel 2D-QW behaviour in Sec $\mathrm{VB}$

We pay attention to another analytical solution [9] in order to introduce the concept of Fourier analysis. The evolution operator has a more concise form in $k$-space (Fourier space) than in $x$-space (real space), so the initial wave function may be transformed to and evolved in $k$ space. By inverse Fourier transformation, the real-space wave functions for the $|\uparrow\rangle$ and $|\downarrow\rangle$ internal states are

$$
\begin{aligned}
& \psi_{\uparrow}(x, t)=\frac{1}{2 \pi} \int_{-\pi}^{\pi} d k e^{-i k x} \frac{e^{-i \omega_{k} t}\left(\sqrt{1+\cos ^{2} k}+\cos k+i e^{-i k}\right)+e^{i\left(\omega_{k}-\pi\right) t}\left(\sqrt{1+\cos ^{2} k}-\cos k-i e^{-i k}\right)}{2 \sqrt{2} \sqrt{1+\cos ^{2} k}}, \\
& \psi_{\downarrow}(x, t)=\frac{1}{2 \pi} \int_{-\pi}^{\pi} d k e^{-i k x} \frac{i\left[e^{-i \omega_{k} t}\left(\sqrt{1+\cos ^{2} k}-\cos k-i e^{i k}\right)+e^{i\left(\omega_{k}-\pi\right) t}\left(\sqrt{1+\cos ^{2} k}+\cos k+i e^{i k}\right)\right]}{2 \sqrt{2} \sqrt{1+\cos ^{2} k}},
\end{aligned}
$$

and finally the probability of finding the walker at a given position $x$ after a walk of $t$ steps is given by

$$
P(x, t)=\left|\psi_{\uparrow}(x, t)\right|^{2}+\left|\psi_{\downarrow}(x, t)\right|^{2} .
$$

Eqs. (7) and (8) contain no dynamics, because the term $e^{-i \omega_{k} t}$ provides no more than a compact notation for the combination of $N$ with $k$, the spatial Fourier variable conjugate to the actual walker displacement $x$.

A numerical calculation of the exact analytical solution, contained in Eqs. (77) and (8), is shown as the red curves and points in Fig. 3, where we compute the probability distribution in both real space [Fig. 3(a)] and Fourier space [Fig. 3(b)] for $N=100$ and 1000. The blue curves show our numerical calculations based on method in Eqs. (6) with the initial state specified in Eq. (5). The results are identical up to a relative error of $10^{-5}$ caused by the numerical integration of Eq. (7). We comment in detail on the forms of these distributions in Sec. III.

The importance of the values $\pm N / \sqrt{2}$, noted in Sec. IIB, is clearly evident in Fig. 3(a), and it was deduced in Ref. 28] that the limiting distribution is concentrated in the interval $\left[-\frac{N}{\sqrt{2}}, \frac{N}{\sqrt{2}}\right]$ as $N \rightarrow \infty$. We will qualify this statement in Sec. IIIA. Although the analytical solution [9, 28] gives the exact probabilities for any position and number of steps, in fact the expressions in Eq. (7), which require numerical integration over complex quantities, are not easy to compute when $N$ becomes large. In this regime, direct numerical calculation of the probability distribution is more straightforward, and we use this approach in Sec. III to reveal the properties and structure of the 1D QW at large $N$.

\section{NUMERICAL STUDIES OF THE 1D QW}

We now consider in detail the properties of the 1D QW introduced in Sec. II. The probability distribution obtained from this quantum algorithm has a number of very striking properties in both real and Fourier space (Fig. (3). Among them are the clear importance of $\pm N / \sqrt{2}$ noted above, the twin-peaked "envelope function" of the $P(x)$ distributions with its remarkable zone of destructive interference around $x=0$, the width of these peaks, and the rapid oscillation of the functions (both $P(x)$ and $F(k)$ ) at high spatial frequencies within the envelope. We clarify immediately that this oscillatory behavior is "real" in the sense that Figs. 2 and 3 show only the probabilities at even steps $0, \pm 2, \pm 4, \ldots$, with the zeroprobability odd steps not shown; the QRW contains additional oscillations in space between the "microscale" of individual steps and the "macroscale" of the walk length. 

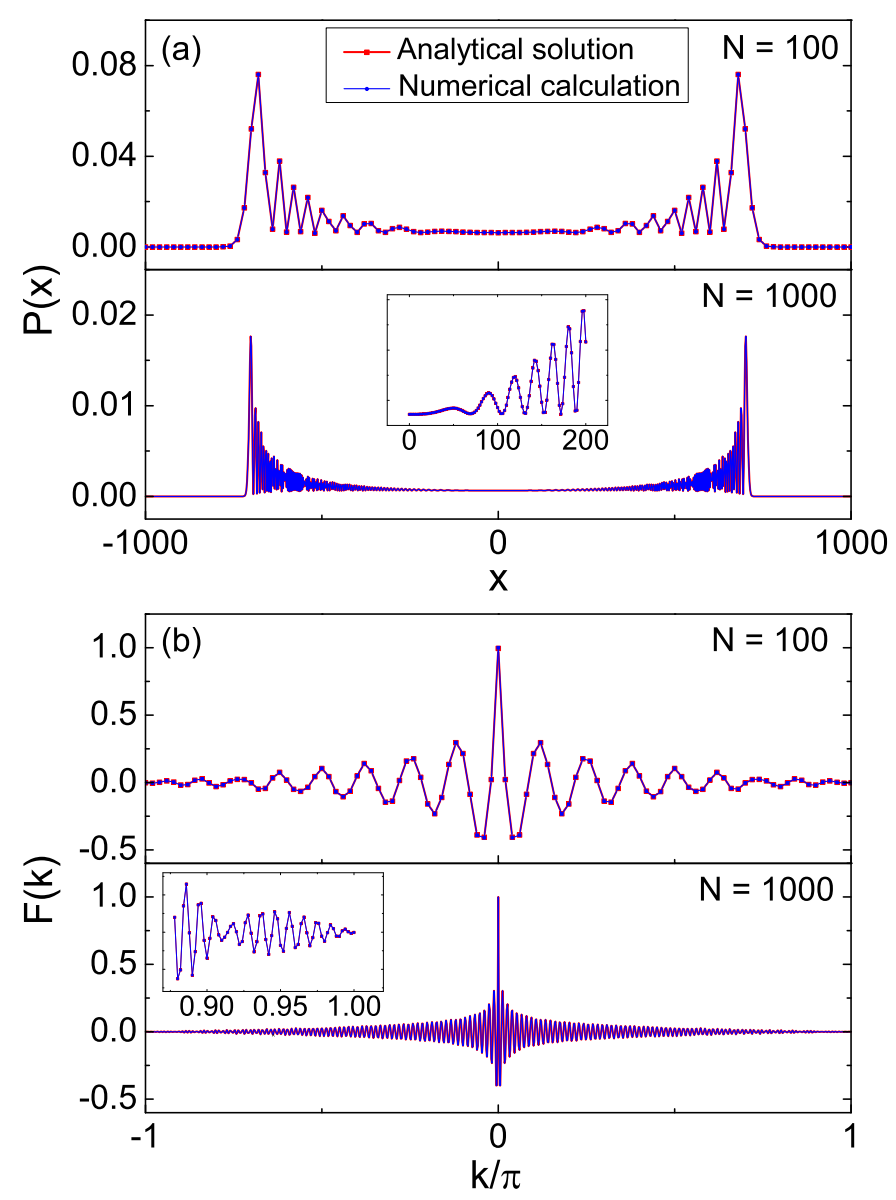

FIG. 3. (color online) Probability distribution for the 1D QW, comparing the exact expression of Eqs. (7) and (8), shown in red, with numerical calculations based on Eqs. (5) and (6) shown in blue, for walks of $N=100$ and 1000 steps. (a) Probability distribution $P(x)$ in real space. (b) Fourier components $F(k)$ of $P(x)$. Insets for $N=1000$ show (a) the probability oscillations near $x=0$ and (b) beats in the Fourier envelope near $k=\pi$ (see text).

\section{A. $N / \sqrt{2}$ Property}

We begin by analyzing the most obvious feature of the QW, which is the tendency for the probability distribution to peak around $0.7 N$. From Sec. IIC it is clear that the factor $1 / \sqrt{2}$ plays an important role in the analytic solution and in physical terms it would appear to mark the crossover in behavior from a low probability arising due to destructive interference to a low probability arising simply from the extremely low likelihood of having more than $85 \%$ of the steps of an unbiased random walk be in the same direction. In Fig. 4 we show numerical results for the probability distributions of $1 \mathrm{D}$ QWs with four different values of $N$, with the position axis normalized by $N$. As $N$ increases, the distributions exhibit both increasingly oscillatory behavior, which we analyze in Sec. IIIB, and a peaking of the envelope function, which becomes sharper as it converges towards a maxi-

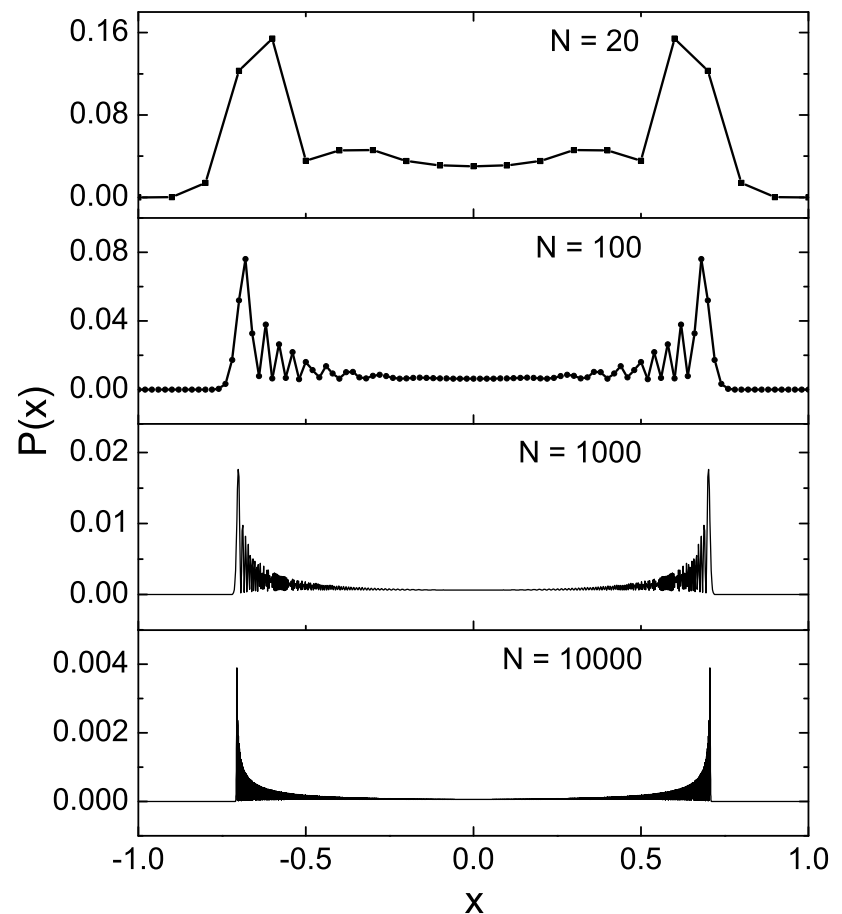

FIG. 4. Numerical results for the 1D QW with different numbers $N$ of steps, scaled to the same horizontal axis. Probabilities are shown only for even values of the site position $x$, with zero values on odd sites excluded from the curves.

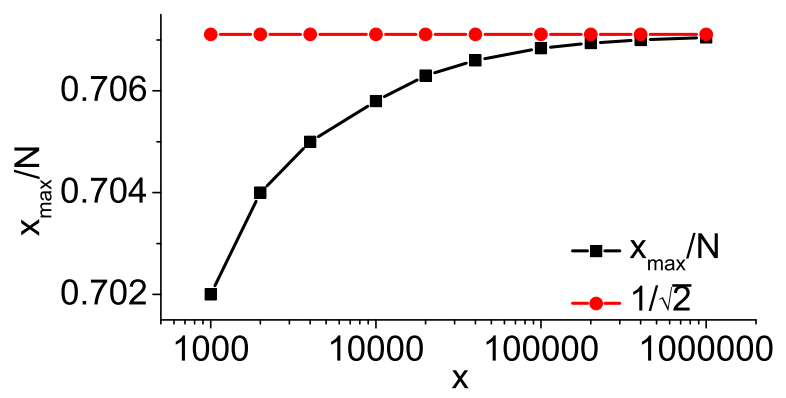

FIG. 5. (color online) Scaling of the position $x_{\max }$ for maximum probability with total step number $N$ in the $1 \mathrm{D}$ QW.

mum probability close to step $N / \sqrt{2}$ (Sec. IIIC).

This convergence in shown in more detail for values up to $N=1000000$ in Fig. 5. The macroscopic feature is indeed a convergence towards $N / \sqrt{2}$. Further, on a relative scale the distribution appears to tend towards $\delta$ functions centered at $\pm N / \sqrt{2}$. However, we caution that this is not the complete story and we consider the asymmetric envelope shape in Sec. IIIC. The point of maximum probability is in fact $x_{\max }=70684$ for $N=100000$ and $x_{\max }=707050$ for $N=1000000$, while the exact value of $1 / \sqrt{2}$ is 0.707106 . Thus in fact there are still more than 25 even steps of finite probability separating $x_{\max }$ from $N / \sqrt{2}$ for $N=1000000$ [see Figs. Z7(i) and 7(j)]. The probability at $x=N / \sqrt{2}$, shown in Fig. 6, is 


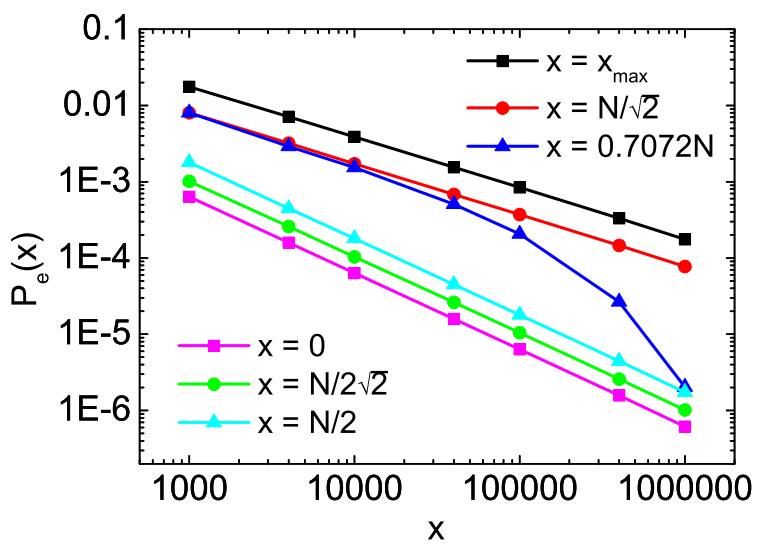

FIG. 6. Representative probability data from different points on the distribution, $x=0, x=N / 2 \sqrt{2}, x=N / 2, x=x_{\max }$, $x=N / \sqrt{2}$, and $x=0.7072 N$, for $1 \mathrm{D}$ QWs of step lengths from $N=1000$ to $N=1000000$. Where relevant, all data are taken from the upper envelope of the distribution.

always close to one half of $P\left(x_{\max }\right)$, and this point marks the approximate crossover where $P(x, N)$ changes from algebraic to exponential decay with $N$ (below). There are always points of finite $P(x)$ beyond $x=N / \sqrt{2}$ and the more exact statement of the result of Ref. [28] is that the normalized support converges to the interval $\left[-\frac{1}{\sqrt{2}}, \frac{1}{\sqrt{2}}\right]$ as $N \rightarrow \infty$. The probability of a walker passing beyond this interval vanishes more rapidly than interference effects can cause it to grow, and the net consequence of the destructive interference between paths is to "pile up" the probability close to (but mostly below) the limits of the interval.

Further insight into the nature of the (upper) envelope function may be obtained by considering the probabilities at different representative positions on the normalized $x$-axis, as shown in Fig. 6. The probabilities $P_{N}(0)$, $P_{N}(N / 2 \sqrt{2})$, and $P_{N}(N / 2)$ all fall linearly with $1 / N$, suggesting a constant weight if binned into intervals whose width scales with $N$. It is worth noting that the weight at position zero, which has the maximum number of interfering paths, does not vanish completely due to destructive interference in any finite-length QW. By contrast, the probability at positions $x=x_{\max }$ and $x=N / \sqrt{2}$ scales as $P \propto N^{-2 / 3}$ [specifically, $P\left(x_{\max }\right)=1.8 N^{-2 / 3}$ and $\left.P(N / \sqrt{2})=0.44 P\left(x_{\max }\right)\right]$, accounting for the sharpening of the distribution peaks with increasing $N$. We return to the question of the peak shape in Sec. IIIC. As noted in the preceding paragraph, beyond $x=N / \sqrt{2}$ there is a very abrupt change in the form of $P(N)$ to an exponential decay, as shown in Fig. [6 for the point $x=0.7072 \mathrm{~N}$ and in the sudden loss of oscillatory behavior in Figs. $7(\mathrm{i})$ and $7(\mathrm{j})$; this we will also analyze in more detail in Sec. IIIC.

\section{B. Oscillatory Behavior}

We turn next to the question of the oscillatory behavior of the probability distribution within its envelope function. We stress again that this has nothing to do with the period- 2 oscillation created by the fact that walkers alternate between odd and even sites at successive steps of the walk. We begin by showing in Fig. 7 the qualitative nature of the oscillations in the real-space probability distribution function for selected regions of the interval, using different values of $N$ to highlight their universal nature.

One of the most important properties of the oscillations is that their effective wavelength, in terms of the fundamental step size, appears to decrease towards larger values of $x$. At the center of the distribution, as shown in Figs. 7(a) and 7(b), they have relatively long wavelengths, but towards the edges these become shorter [Figs. 7(c) and 7(d)] until in the region $x \approx 0.6 N$ they have only twice the fundamental length [Figs. 7](g) and $7(\mathrm{~h})]$. Towards the center of each half of the distribution, some beat-like structures develop [Figs. 7(e) and $7(f)]$. As the peak is approached, the frequencies drop and the oscillations vanish suddenly at $x=N / \sqrt{2}$ [Sec. IIIA, Figs. 7(i) and 7(j)]. We draw attention to the fact that the distribution also has an effective lower envelope function, in that the probability is never zero on any even points and in fact is very much larger than the size of the oscillations close to $x=0$ [Figs. 7(a) and $7(\mathrm{~b})$ ], but we do not analyze this further here.

To quantify the nature of the oscillations, we count the numbers of peaks in the probability distribution within different intervals and for QWs of different $N$. First of all, by counting the total number of peaks it is clear (Fig. 8) that this is linearly proportional to (approximately $8.5 \%$ of) $N$. Subdividing the QW into intervals of fixed length and counting the peaks in each of these gives the results shown in Table I. By comparing these peak counts horizontally, meaning for different intervals within walks of the same $N$, a steady increase in frequency becomes apparent out to $x \approx 0.6 N$, from very long-wavelength (periods of 50 or more steps) oscillations near $x=0$ to extremely rapid (period-4) ones when $x$ is a signifcant fraction of $1 / \sqrt{2}$. We remind the reader here that a period of 4 in a system where odd sites have probability zero is essentially a max-min-max-min-... structure within the envelope [Figs. $7(\mathrm{~g})$ and $7(\mathrm{~h})]$. Thus spatial information about the QW is truly contained on all length scales. By comparing the peak counts vertically, meaning for different values of $N$, it becomes apparent that corresponding regions have precisely the same frequencies, with the maximum frequency occurring in the region around $x=0.58 N$. Thus the spatial modulation of the QW is a quantity independent of $N$; although QWs of different $N$ cannot be called self-similar, they do share similarities in particular aspects.

Other forms of similarity and scaling appear in particular segments of the probability distribution. Considering 
TABLE I. Numbers of peaks in the probability distribution $P(x)$ found within windows of width 100 steps for QWs of $N=1000$, 10000 , and 100000 .

\begin{tabular}{|c|c|c|c|c|c|c|c|}
\hline interval $(N=1000)$ & {$[0,100]$} & {$[100,200]$} & {$[200,300]$} & {$[300,400]$} & {$[400,500]$} & {$[500,600]$} & {$[600,700]$} \\
\hline number of peaks & 2 & 5 & 8 & 12 & 17 & 23 & 17 \\
\hline interval $(N=10000)$ & {$[500,600]$} & {$[1500,1600]$} & {$[2500,2600]$} & {$[3500,3600]$} & {$[4500,4600]$} & {$[5500,5600]$} & {$[6500,6600]$} \\
\hline number of peaks & 2 & 5 & 8 & 12 & 17 & 23 & 17 \\
\hline interval $(N=100000)$ & {$[500,600]$} & {$[15500,15600]$} & {$[25500,25600]$} & {$[35500,35600]$} & {$[45500,45600]$} & {$[55500,55600]$} & {$[65500,65600]$} \\
\hline number of peaks & 2 & 5 & 8 & 12 & 17 & 23 & 16 \\
\hline interval $(N=10000)$ & {$[5000,5100]$} & {$[5500,5600]$} & {$[5600,5700]$} & {$[5700,5800]$} & {$[5800,5900]$} & {$[5900,6000]$} & {$[6000,6100]$} \\
\hline number of peaks & 20 & 23 & 24 & 25 & 25 & 24 & 23 \\
\hline interval $(N=100000)$ & {$[50000,50100]$} & {$[50000,50200]$} & {$[52000,52100]$} & {$[54000,54100]$} & {$[56000,56100]$} & {$[58000,58100]$} & {$[60000,60100]$} \\
\hline number of peaks & 20 & 20 & 21 & 22 & 24 & 25 & 23 \\
\hline
\end{tabular}

first the region close to $x=0$, Fig. 7 (a) shows 15 peaks in the region $[0,300]$ for $N=1000$. The corresponding region for $N=10000$, which is $[0,3000]$, contains 148 peaks, confirming the conclusion drawn above that the frequencies are the same in corresponding areas for differing $N$, leading to the overall linearity in $N$ of the peak number (Fig. 8). However, by counting the first 15 peaks in the probability distribution for $N=10000$, shown in Fig. 7 (b), they fill the interval $[0,960]$, indicating a $\sqrt{N}$ scaling of the maximum wavelengths around the center of the distribution. We clarify that this is not in contradiction with Table I, where the representative low- $x$ intervals are taken at different finite values of $x$.

This type of scaling may also be observed in other regions of the QW probability distribution. Focusing next on the special structures noted above, Figs. 7(c) and Figs. 7(d) show three of these, which we find quite reproducibly in the region around $x=N / 2$. More accurately, these macroscopic dips of the distribution envelope appear around $x=0.36 N, x=0.45 N$, and $x=0.58 N$. The interval around $0.58 \mathrm{~N}$, which is also the region with maximum oscillation frequency, shows a particularly remarkable beating structure [Figs. 7(e) and 7(f)], with multiple points where the upper and lower envelopes meet. Figures $7(\mathrm{~g})$ and $7(\mathrm{~h})$ show the maximal frequency regime and parts of the beating envelopes in the fullest detail, and Table II contains the corresponding information for the beating interval for QWs of $N=1000,10000$, and 100000. Again it is clear that a factor-10 increase in $N$ causes only a factor $\sqrt{10}$ magnification of the width of the beating structure and, given the fixed maximal frequency in this interval, of the number of peaks it contains.

We close our discussion of the scaling of peak widths by considering the probability oscillations around $x=$ $N / \sqrt{2}$. By counting the width of the region covered by

TABLE II. Position, width, and number of peaks in the distinctive beating structure around $x=0.58 \mathrm{~N}$ for walks of $N=1000,10000$, and 100000 steps.

\begin{tabular}{|c|c|c|c|}
\hline $\mathrm{N}$ & region & width & number of peaks \\
\hline 1000 & {$[546,604]$} & 58 & 14 \\
\hline 10000 & {$[5682,5860]$} & 176 & 44 \\
\hline 100000 & {$[57452,58016]$} & 566 & 141 \\
\hline
\end{tabular}

the last 10 peaks up to and including the peak of maximum probability, we find that this scales according to $N^{1 / 3}$ (Fig. 9). Similarly, the full width at half maximum height (FWHM) of the leading peak in $P(x)$ also scales with $N^{1 / 3}$, and hence this last peak retains its aspect ratio when $x$ is normalized by $N$. Thus we demonstrate the presence of algebraic scaling in the probability oscillations over the full distribution.

\section{Peak Shape}

Next we consider the shape and scaling of the asymmetric envelope of peaks in the probability distribution to ascertain its functional form, $P(x, N)$. The dependence on $N$ is largely contained in Fig. 6 and we make these results more systematic here. Concerning the dependence on $x$, we take the results of Sec. IIIA as a demonstration that the "crossover" region just beyond the maximum peak becomes a set of vanishing measure at large $N$; to fit the envelope function, meaning the set of points extracted from the full data set that fall close to the upper edge of $P(x)$, for the region $x \leq x_{\max }$, we assume that it diverges at $x= \pm N / \sqrt{2}$ for large values of $N$. Before considering the QW, we recall that the functional form of the probability distribution for a classical random walk (Sec. IIA) becomes a Gaussian at large $N$, with the form

$$
P(x)=P_{0}+A e^{-\frac{(x-b)^{2}}{2 \sigma^{2}}},
$$

where $\sigma=\sqrt{N}, A=1 / \sqrt{2 \pi N}$, and $P_{0}=0=b$ for a normalized and centered distribution. This is an exponential function whose characteristic width scales with $\sqrt{N}$.

By contrast, for the QW with large $N$ we find an excellent fit to an algebraic form,

$$
P_{e}(x)=P_{0}+a(b-x)^{-c}
$$

for each half of the distribution, with $b= \pm N / \sqrt{2}$. To quantify the extent of the validity of such a fit, we examine the data on logarithmic axes [inset, Fig. [10(a)], finding that a single power-law provides a robust description of the envelope for the entire region $0.4 N \leq x \leq x_{\max }$. For the three free parameters, our results as $N \rightarrow \infty$ 

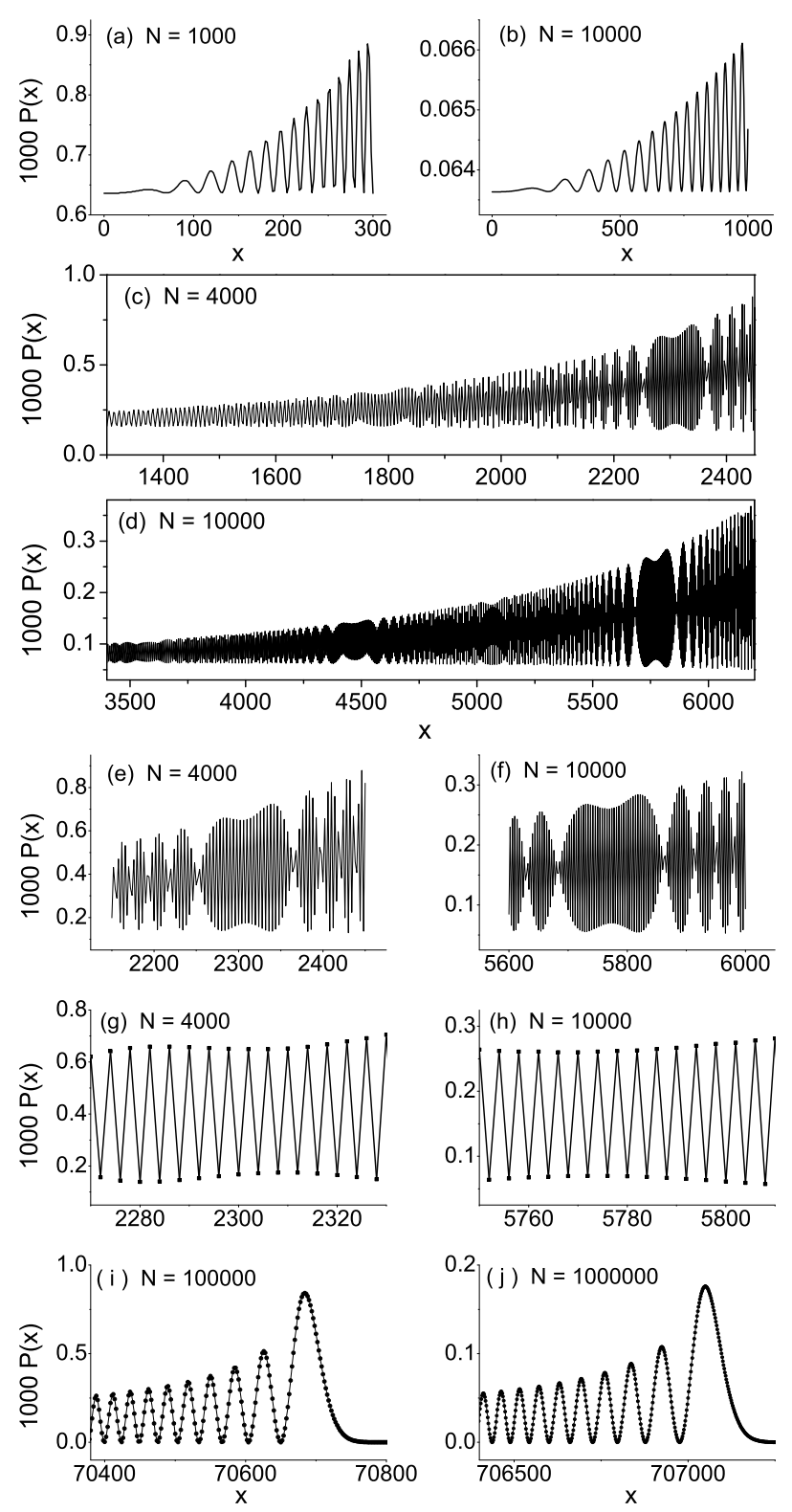

FIG. 7. Illustration of probability distributions in different parts of the interval for $1 \mathrm{D}$ QWs of different step numbers $N$. First 15 oscillation peaks for (a) $N=1000$ and (b) $N=$ 10000. Central region of the distribution for (c) $N=4000$ and (d) $N=10000$, showing apparent beating. Detail of the envelopes in the region of the beating structure for (e) $N=$ 4000 and (f) $N=10000$. Detail within the envelope of the beat structure for (g) $N=4000$ and (h) $N=10000$, showing the most rapid oscillations in the distribution. Probability maxima for (i) $N=100000$ and (j) $N=1000000$.

(in practice, up to $N=1000000$ ) indicate that the constant $P_{0}=-B / N$ with $B=1.884$. Fits performed using only two remaining free parameters, and illustrated in the main panel of Fig. 10(a) for the case $N=1000000$, allow us to deduce for the large- $N$ limit that the prefactor approaches $a=A / \sqrt{N}$ with $A=1.5$ and the exponent approaches $c=0.5$. We conclude that to an excellent ap- proximation the envelope function follows a square-root form in $x$ measured away from $x= \pm N / \sqrt{2}$, and this determines the algebraic form of the peak width. The behavior of the prefactor $A$ ensures that $P_{e}(x) \propto 1 / N$ across all of this range, consistent with the results of Fig. [6 but excluding the final peak. We remind the reader that the envelope function $P_{e}(x)$ is not a quantity obeying a normalization law as the true distribution $P(x)$ does.

Below $x=0.4 N$, the shape of the envelope begins to deviate from the universal square-root form [inset, Fig. 10(a)]. To ascertain its shape close to the center of the distribution, we instead apply a fit of the form

$$
P_{e}(x)=P_{0}+a^{\prime} x^{c^{\prime}}
$$

for each half of the distribution. Again the data on logarithmic axes [inset, Fig. 10(b)] show an excellent fit to a single set of parameters over a broad region, $0 \leq x \leq 0.2 N$, where $P_{0}=B^{\prime} / N$ with $B^{\prime}=0.615$, $a^{\prime}=A^{\prime} / N^{c^{\prime}+1}$ with $A^{\prime}=1$, and $c^{\prime}=2$. The results are shown in the main panel of Fig. 10(b). Thus the $P \propto 1 / N$ form is maintained, the universal behavior of the envelope around its center is a quadratic dependence on $x$, and there is a relatively significant constant contribution that reflects directly the incomplete nature of destructive interference in the central region of the $\mathrm{QW}$. We regard the intermediate regime $0.2 N \leq x \leq 0.4 N$ as a crossover zone between the two limiting forms [Eqs. (10) and (11)] and do not consider it further.

We close our discussion of the probability distributon $P(x, N)$ by considering the region $x>N / \sqrt{2}$. Here there are no longer any oscillations [Figs. $7(\mathrm{i})$ and $7(\mathrm{j})]$ and $P(x, N)$ is the "envelope." In Sec. IIIA [Fig. 6] we showed that the dependence on $N$ crosses very rapidly to an exponential decay around $x=N / \sqrt{2}$. A complete fit of the data in this regime reveals the form

$$
P(x)=P_{0}+a e^{-d \frac{(x-b)^{1.5}}{N^{0.5}}}
$$

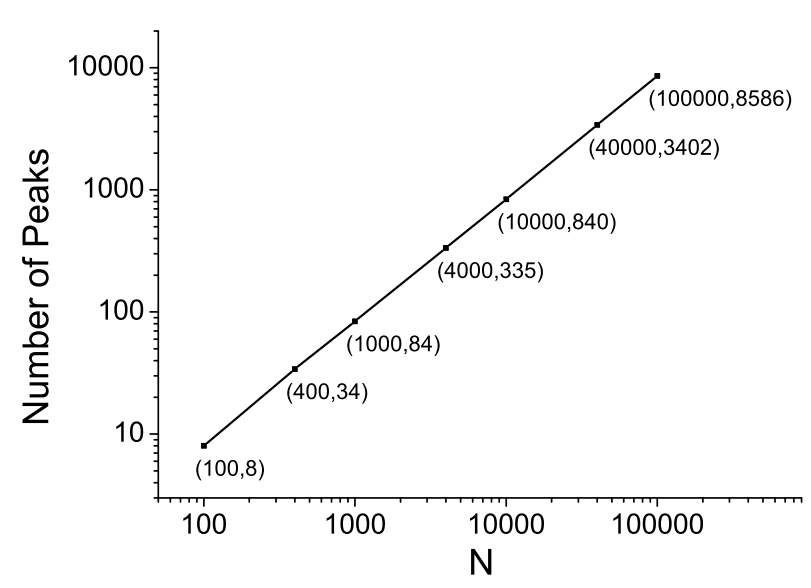

FIG. 8. Total number of peaks in the probability distribution for QWs from $N=100$ to 100000 . 


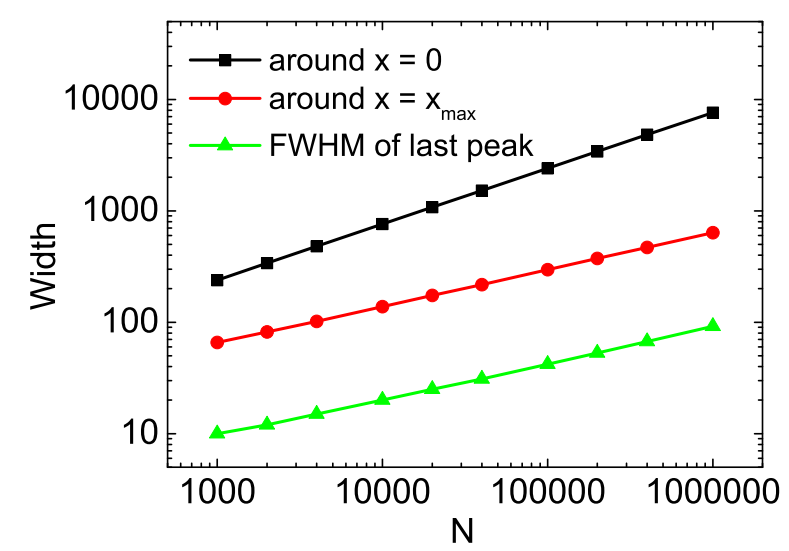

FIG. 9. Width in $x$ of different numbers of peaks in the probability distribution for QWs over the full range of $N$ values studied. The width of the first 10 peaks from $x=0$ (black) scales with $\sqrt{N}$; the width of the last 10 peaks up to the maximum (red) and also the FWHM of the tallest peak (green) both scale with $N^{1 / 3}$.

with $P_{0}=0, b=N / \sqrt{2}, d=3.2$, and $a=A N^{-2 / 3}$ with $A=0.8$, i.e. an exponential and pseudo-Gaussian behavior but with unconventional alterations to the exponents in both $x$ and $N$. The effectiveness of this fit is shown in Fig. 10(c), which also highlights how rapidly the probability falls away in a short distance beyond $x=N / \sqrt{2}$.

\section{Fourier Transformation of the 1D-QW}

In Sec. IIIB we explored the rich spatial frequency information contained in the oscillations of the probability distribution $P(x)$. The QW contains oscillations on all length scales from ultra-short wavelengths around $x=0.58 N$ to long-wavelength oscillations scaling as $\sqrt{N}$ around $x=0$, with similar algebraic scaling around $x=x_{\max }$. These differing spatial frequencies can even combine to create highly reproducible beating structures in certain regions. All of this information should be reflected in the Fourier transform of $P(x)$, which we discussed from an analytical point of view in Sec. IIC.

Here we perform a discrete Fourier transformation on the data sets for 1D QWs of all lengths $N$, finding results for the Fourier components, $F(k)$, of the type shown already in Fig. 3(b) for $N=100$ and $N=1000$. $F(k)$ possesses a primary peak with amplitude $F(0)=1$ at $k=0$, flanked by two secondary peaks with negative components at $k \simeq \frac{5 \pi}{N}$, and then shows an oscillatory form between positive and negative values of the Fourier components. The oscillations are again contained within a decaying envelope function, which we find to be identical at positive and negative values, and the $k$-space periodicity of the oscillation is remarkably constant across the range $-\pi<k \leq \pi$. This result is a clear reflection of the fact that spatial information is present in the prob-
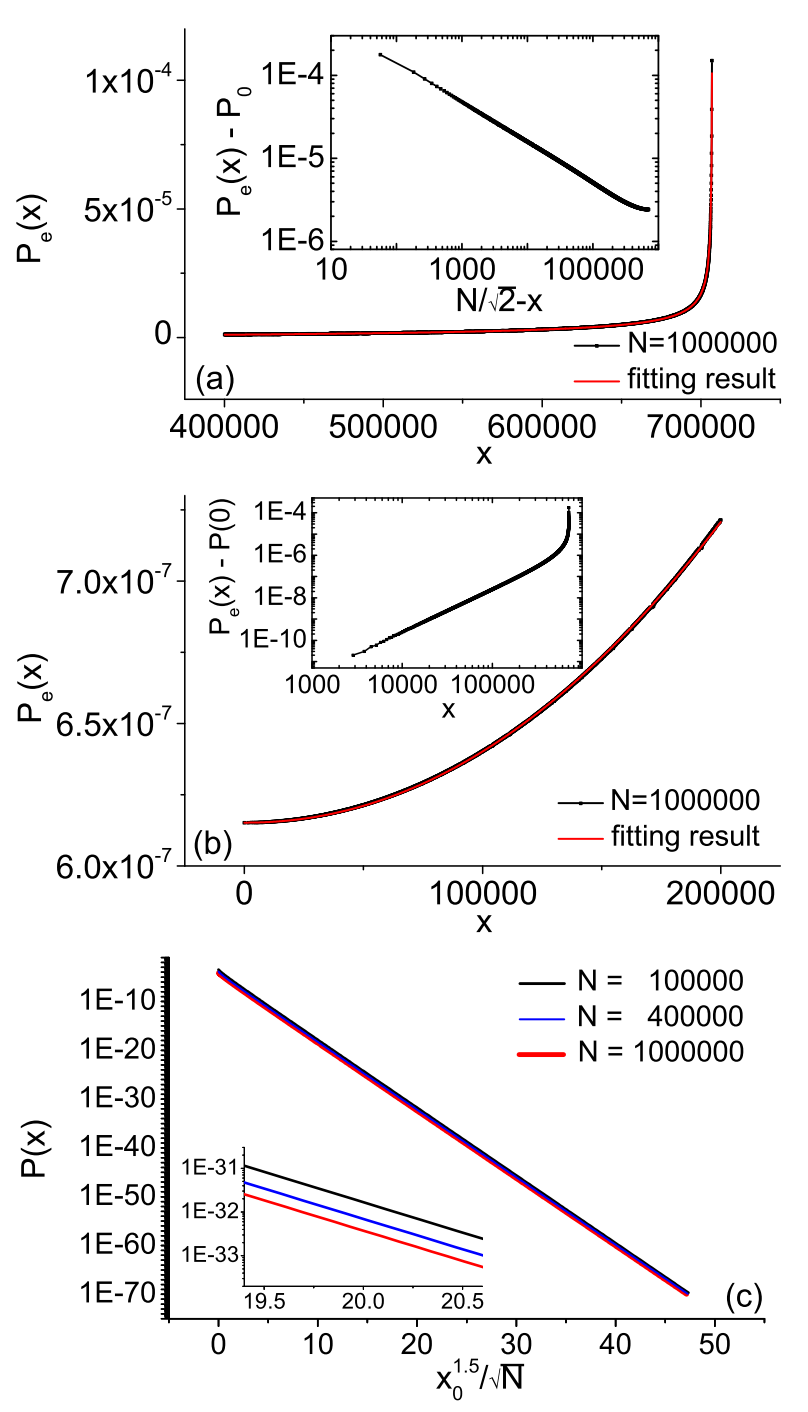

FIG. 10. Fit to the envelope of the probability distribution, $P_{e}(x)$, for a $\mathrm{QW}$ of $N=1000000$ steps. (a) Fit for the interval $400000 \leq x \leq x_{\max }$ using the algebraic form of Eq. (10) with $B=1.884, A=1.487, b=707144$, and $c=0.4918$ (see text). (b) Fit for the interval $0 \leq x \leq 200000$ using the algebraic form of Eq. (11) with $B^{\prime}=0.6152, A^{\prime}=1.0017$, and $c^{\prime}=2.074$ (see text). (c) Fit to the probability distribution $P(x)$ for the interval $x>N / \sqrt{2}$ using the exponential form of Eq. (12) with $P_{0}=0, b=N / \sqrt{2}, d=3.2$, and $A=0.8$ (see text).

ability distribution on all length scales, and the mixture of positive and negative components across the range of $k$ is manifest in complex mixing phenomena such as the beating structure. However, beyond the large $k=0$ component there are no special spatial frequencies appearing in the distribution. We comment here that the constant component $F(0)=1$ is simply the sum of all data in real space, and therefore is the result expected for a normalized distribution.

We begin our quantitative analysis of the Fourier transform $F(k)$ by counting its peaks. Figure 11(a) confirms 

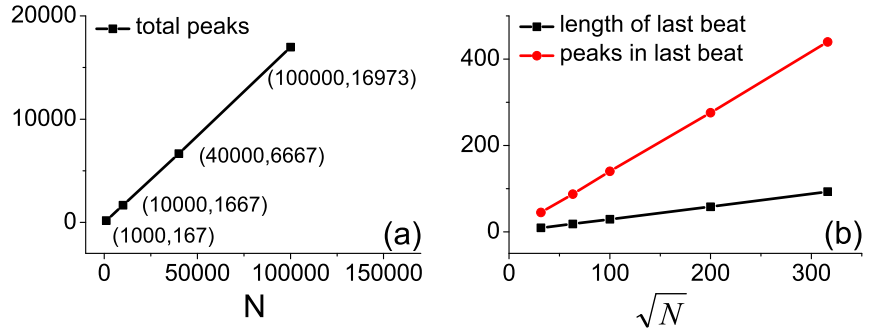

FIG. 11. (color online) (a) Number of peaks in Fourier space for QWs of lengths $N=100$ to 100000. (b) Length of the envelope function of the final beat below $k=\pi$, in units of $N k / 2 \pi$ (black), and the number of peaks in this region (red), shown as a function of $\sqrt{N}$.

that the total number of peaks in the Fourier spectrum scales linearly with $N$, as in real space and again with a constant of proportionality of order $8 \%$ (more precisely, $1 / 12$ ) for each half of the transformed distribution. As in real space, we may also count the peaks in particular parts of the distribution to investigate their scaling form. The most striking feature of the Fourier transformed data for large values of $N$ is a reappearance of beating phenomena between the upper and lower envelope functions. In complete consistency with the results of Sec. IIIB, where the beating structures were observed in the region of the distribution with the highest spatial frequencies, the Fourier-space beats are clearest close to $k=\pi$. In Fig. 12 we illustrate this property with the $k$-axis rescaled to $N k / 2 \pi$ to better reflect the number of Fourier components in the data set. As in Sec. IIIB, we may characterize the structure of the beat pattern by considering the length of the final beat and the number of peaks it contains, which are tabulated in Table III and illustrated in Fig. 11(b). From the latter it is clear once again that the beat structures scale according to $\sqrt{N}$.

Proceeding as in Sec. IIIC, we consider the possibility of a universal fit to the envelope function $F_{e}(k, N)$, whose $k$-dependence is valid for all values of $N$. The $N$ dependence of $F_{e}(k, N)$ is shown in Fig. 13)(a) for values of $k$ from across the full range, and the constant slope gives the clear result $F_{e}(k, N) \propto 1 / \sqrt{N}$. Mindful of the fact that the real-space envelope changes form between the limits of small and large $x$, we consider the functional

TABLE III. Characterization of oscillation frequencies in Fourier space. The length of the last beat is quoted in units where the interval of the Fourier components is rescaled to $(-N k / 2 \pi, N k / 2 \pi]$.

\begin{tabular}{|c|c|c|c|}
\hline $\mathrm{N}$ & total peaks & peaks in last beat & length of last beat \\
\hline 1000 & 167 & 9 & 45 \\
\hline 4000 & 667 & 18 & 87 \\
\hline 10000 & 1667 & 29 & 140 \\
\hline 40000 & 6667 & 58 & 276 \\
\hline
\end{tabular}
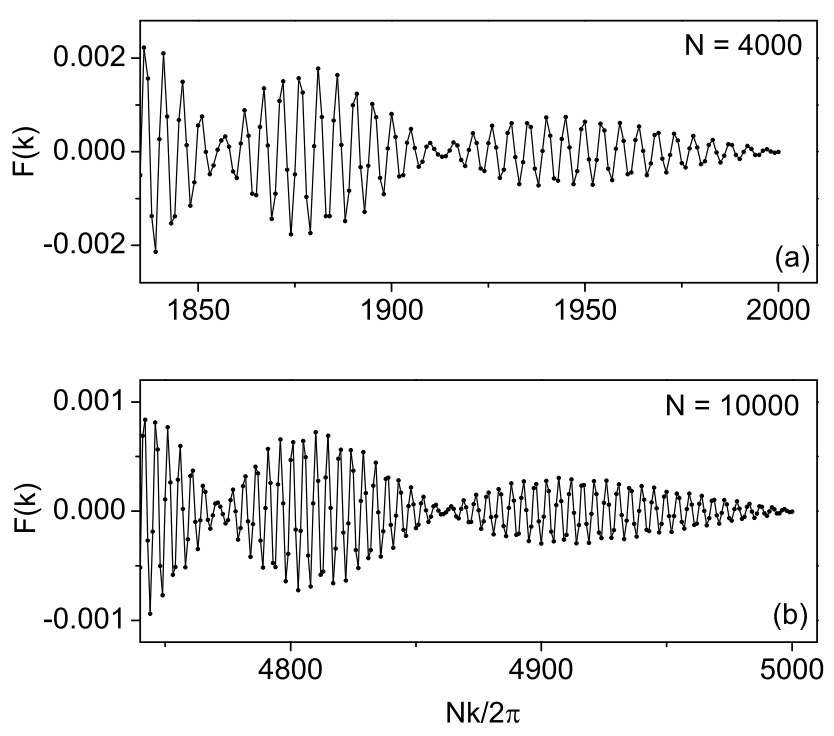

FIG. 12. Fourier components of the probability distribution close the maximum frequency, shown rescaled to $N k / 2 \pi$. In this regime the envelope function shows clear beating behavior.

forms

$$
F(k)=\frac{A}{\sqrt{N}}\left(\frac{k}{\pi}\right)^{-c}
$$

and

$$
F(k)=\frac{A^{\prime}}{\sqrt{N}}\left(1-\frac{k}{\pi}\right)^{c^{\prime}}
$$

for small and large $k$. As shown in Figs. 13(b) and 13(c), Eq. (13) with exponent $c=1 / 2$ provides an excellent fit at small $k$, out to $k \approx 0.2 \pi$, and Eq. (14) with $c^{\prime}=1$ an excellent fit for all $k$ values in the upper half of the range. Thus we find the $k$-dependence of the envelope of Fourier components to be algebraic over the whole range, with an inverse square-root decay away from $k=0$ crossing over to a linear decrease as $k$ approaches $\pi$.

In summary, the Fourier transform of the QW probability distribution contains all of the same information in a complementary form. It is bounded by upper and lower envelope functions with the same algebraic decay. It demonstrates that spatial frequencies are present on all scales from the inverse step length to the inverse system size, with no special internal period(s) but with distinctive beating structures on a length scale of $\sqrt{N}$. While the QW does not satisfy the strict definition of selfsimilarity (no fractal structures appear), many of its features are similar and scale-invariant across the full range of $N$ values. Thus the simple quantum evolution algorithm of Sec. IIB contains a very rich variety of spatial information. 

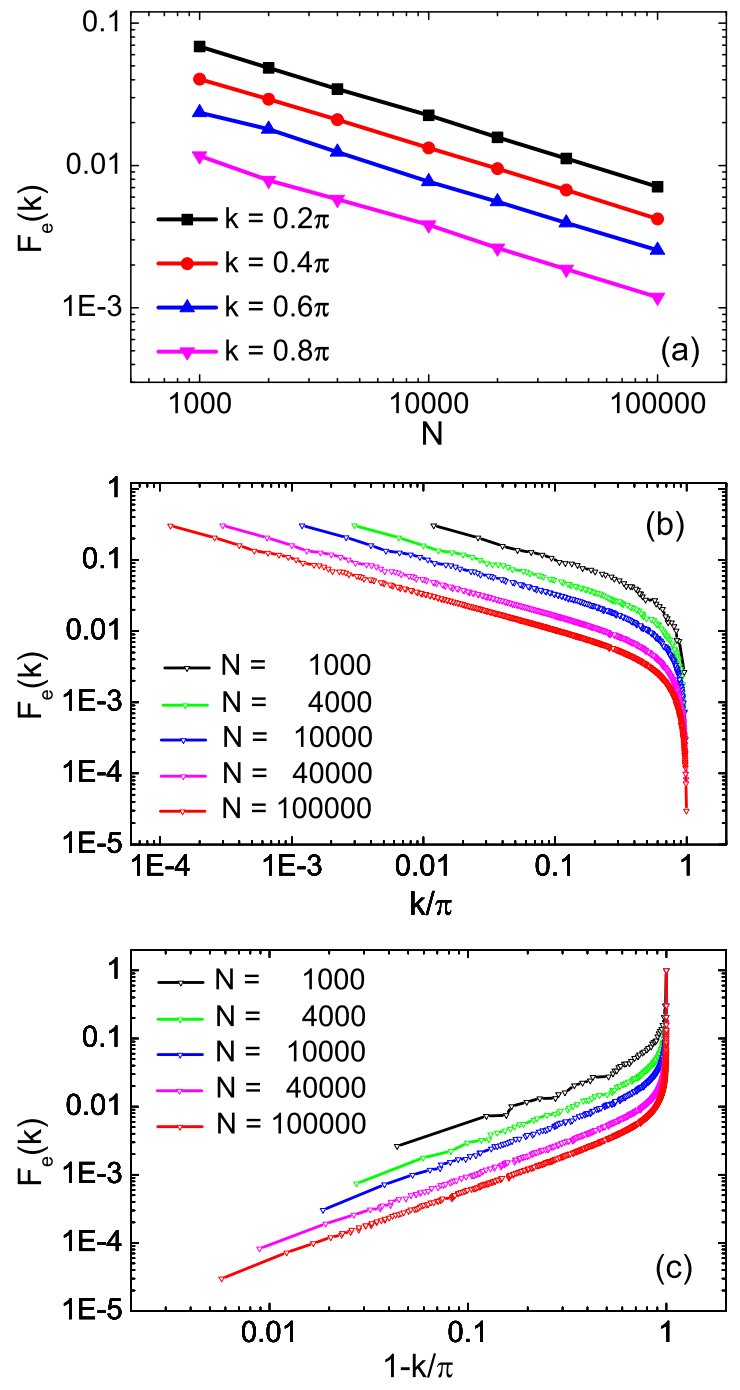

FIG. 13. Fit to the envelope of the Fourier transform of the probability distribution, $F_{e}(k)$, for QWs of $N=1000$ to 100000 steps. (a) $N$-dependence of $F_{e}(k)$ for four different values of $k$, showing a clear algebraic decay with exponent $1 / 2$. (b) Fit using the algebraic form of Eq. (13), demonstrating its relevance for the interval $0 \leq k \leq 0.2 \pi$ and returning the exponent $c=1 / 2$. (c) Fit using the algebraic form of Eq. (14), demonstrating its relevance for the interval $0.4 \pi \leq k \leq \pi$ and returning the exponent $c^{\prime}=1$.

\section{TWO-DIMENSIONAL QUANTUM WALKS}

\section{A. Classical Random Walk in 2D}

As noted in Sec. I, it is the advent of experiments capable of realizing a 2D QW [26] that has caused the resurgence of interest, both experimental and theoretical, in the field. Before discussing the range of properties exhibited by QWs in $2 \mathrm{D}$, it is helpful to review the classical case. In principle there are two ways to generalize the unbiased 1D classical random walk to 2D.
1) Adopting a square grid, the walker is equipped only with a two-face coin and therefore flips it once to step to $(x \pm 1, y)$, then a second time to arrive at $(x \pm 1, y \pm 1)$. Now the conventional $N$-step 1D binomial distribution for $x$ or $y$ is recovered by summing over the probabilities in the orthogonal direction and it is easy to show for any $N$ that $P(x, y)=P(x) P(y)$. In the large- $N$ limit, the distribution approaches

$$
P(x, y)=\frac{1}{2 \pi N} e^{-\frac{x^{2}+y^{2}}{2 N}}
$$

and the mean distance of the walker from the origin after N steps is $\langle r\rangle=\sqrt{N}$.

2 ) Remaining on a square grid, the walker at site $(x, y)$ has equal probabilities of $1 / 4$ (equivalent to a four-face coin) to move to any of the points $(x \pm 1, y \pm 1)$. It results in an identical distribution to case (1).

A further generalization is to consider a continuous space in which the walker takes unit-length steps at any random angle $0 \leq \phi<2 \pi$. The walk approaches perfect circular symmetry $\left(r^{2}=x^{2}+y^{2}\right)$ at large values of $N$.

\section{B. Two Types of 2D QW}

With a view to experimental realization, 2D QW also has two-face coin scheme and four-face coin scheme.

A four-face coin $H_{4}$ can be constructed by product the Hadamard operators, i.e. $H_{4}=U_{H} \otimes U_{H}$ (as well as corresponding initial state). It evolves to a distribution shown in Fig. 14 for a walk of 100 steps. We note immediately that this $2 \mathrm{D} \mathrm{QW}$ is a directly expand of $1 \mathrm{D} \mathrm{QW}$, in which $P(x, y)=P(x) P(y)$. One will understand this equivalence by invoking the matrix identity

$$
\left(A_{1} B_{1}\right) \otimes\left(A_{2} B_{2}\right)=\left(A_{1} \otimes A_{2}\right)\left(B_{1} \otimes B_{2}\right),
$$

$A_{1}, A_{2}$ represent the Hadamard operator $U_{H}$ and $B_{1}, B_{2}$ for the initial state $|\uparrow\rangle+i|\downarrow\rangle$.

The two-face-cion scheme lead to a completely different 2D distribution, shown in Fig. 15 for a walk of 100 cycles. Specifically, an evolution protocol using the same two-face coin twice in each complete cycle to generate successive steps in the $x$ and $y$ directions. The first flip of the coin selects the direction of $\pm x$ and the second $\pm y$. A full cycle can be represented as $\hat{U}=S_{y} Y\left(S_{x} Y\right)$ and the quantum state after $N$ evolution cycles as $\left|\psi_{N}\right\rangle=$ $(\hat{U})^{N}\left|\psi_{0}\right\rangle$.This probability distribution has several properties in common with the $1 \mathrm{D} \mathrm{QW}$, including strong probability peaks far from the center, strong destructive interference everywhere near $(x, y)=(0,0)$, and oscillatory behavior around the locus of maxima. However, the qualitative features of this QW are strikingly different from those of the four-face-coin walk, most notably in that the peaks are rotated by $45^{\circ}$, they occur right at the edge of the system, and here the locus of maxima does show some tendencies towards a circular shape, even if the peaks upon it remain strongly anisotropic and fourfold symmetric. The location of the probability maxima 
is really very anomalous, in that the minimum of the destructive interference is truly obtained when a walker makes all $N$ of its steps in the same direction for one lattice orientation, but precisely $N / 2$ in each direction for the other orientation.

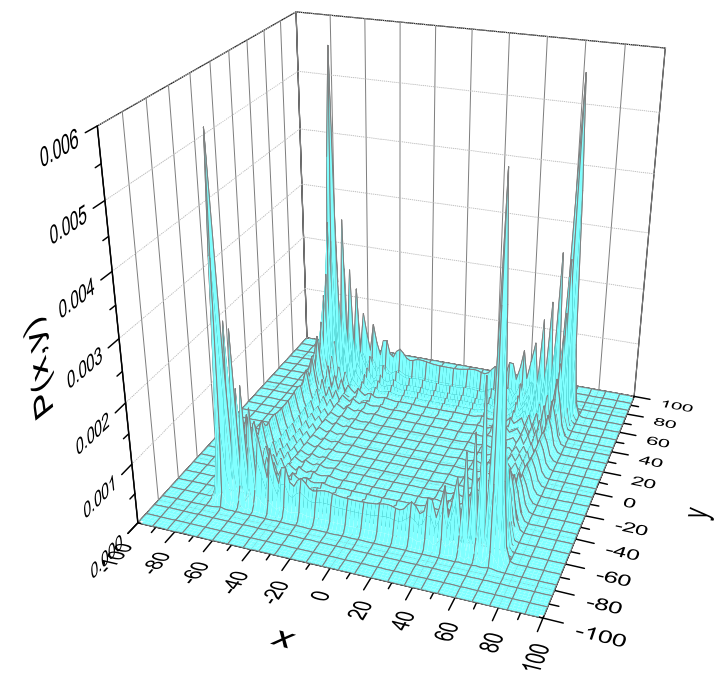

FIG. 14. Probability distribution of 2D QW effected using a four-face coin selecting steps in the $\pm(x+y)$ and $\pm(x-y)$ directions, for $N=100$ steps.

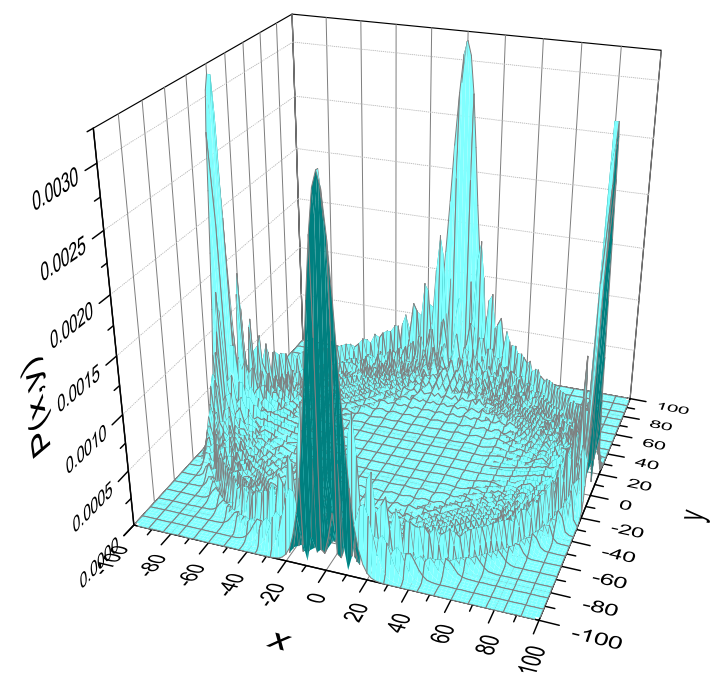

FIG. 15. Probability distribution of 2D QW effected using a two-face coin twice in each cycle to select steps in the $\pm x$ and $\pm y$ directions, for $N=100$ cycles.

Fig. 15 can also be realized by a four-face coin(Grove walk[11]). In one of the most notable recent studies [15, 16], Di Franco and coauthors proved the equivalence of the spatial probability distributions between the 2D QW using a single two-face coin, which these authors termed the "alternate quantum walk" (AQW) and the Grover walk. The AQW is actually a $2 \mathrm{D} \mathrm{QW}$ in which the two direction are maximal entangled.

The Grover coin can be expressed in the form

$$
G_{4}=\frac{1}{2}\left(\begin{array}{cccc}
-1 & 1 & 1 & 1 \\
1 & -1 & 1 & 1 \\
1 & 1 & -1 & 1 \\
1 & 1 & 1 & -1
\end{array}\right)
$$

Noted that the distribution and spreading rates are effected by the initial state [11, 13]. Eq18] is the one providing Grove walk a maximum spread rate

$$
\left|\psi_{0}^{m}\right\rangle=\frac{1}{2}(|\uparrow\rangle-|\rightarrow\rangle-|\leftarrow\rangle+|\downarrow\rangle)\left|0_{x}, 0_{y}\right\rangle .
$$

We conclude here the difference between the two type of $2 \mathrm{D}$ distribution, is not distinguished by two-face coin or four-face coin, but whether the two orthogonal directions are entangled. Fig. 14 can also be realized using two uncorrelated two-face coins. Some authors have studied decoherence [29] and localization 30] in 2D QWs using the concept of two coins, and others have quantified the effects of entanglement by a partial or complete swapping of the two coins after each step of the walk [12].

The method of generating a QW by using the same two-face coin twice, to which we refer henceforth as the AQW [15, 16], is an important one for a number of reasons. First and foremost is that two-state systems are much easier to find, or to produce, in any physical realization of a quantum walker, and hence are much more relevant for experimental implementation. Secondly, this maximally entangled protocol contains further unconventional phenomena, which we discuss in Sec. V. Further, coin entanglement provides a valuable and completely general means of constructing and perhaps also of realizing high-dimensional QWs using only two-face coins.

We conclude this subsection by contrasting the two QWs generated by unentangled and by maximally entangled coins. The unentangled system appears to show a perfectly square, factorized probability distribution (a result we demonstrate in Sec. V) characteristic of the 1D QW, with maxima at positions converging to $( \pm N / \sqrt{2}, \pm N / \sqrt{2})$. The entangled case shows a circular "probability front" and significant complexity in the interference pattern within it. In the sense that the unentangled situation can be discussed as accelerated diffusion from the classical random walk to the quantum walk as a result of destructive interference, so the maximally entangled walk appears to show a still further accelerated diffusion. Indeed, it achieves a situation where information propagates to the very edge of the system with high probability, which is a quite remarkable consequence of near-perfect destructive interference among trajectories in the center region. A possible interpretation of this result may be found in preservation of the information content of the coin, because the degree of two-coin entanglement is "complete" in the sense that the walk can be generated using only one coin. 


\section{NUMERICAL STUDIES OF 2D QWS}

For an exact characterization of the 2D QWs introduced in Sec. IVB, we turn now to a numerical investigation of their probability distributions. We begin by contrasting in Table IV the analytical results for the probabilities of the two walks for $N=6$ steps.

This exercise makes clear that the two QWs are radically different from the outset. For the unentangled case, it is easy to see the result one may already suspect from Fig. 14, that the probability distribution of the unentangled 2D QW, $P(x, y)=P(x) P(y)$, is a direct product of two $1 \mathrm{D}$ QWs in the $x$ and $y$ directions. We will shortly demonstrate it numerically for large values of $N$. We remind the reader that this result is not exactly intuitive, given that the walk steps are each made in the $x \pm y$ direction of the lattice and as such would appear to entangle the two directions completely; however, this result also emerges from the $2 \mathrm{D}$ classical random walk (Sec. IVA). For the entangled case, the probability distribution has no direct relation to the 1D QW and indeed already shows the concentration of probability along the $x$ and $y$ directions rather than along the diagonals.

\section{A. Cross-Sections of the Probability Distribution}

For our numerical calculation of the characteristics of the different 2D QWs, we have computed the probability distributions for both walks up to $N=1000$ steps, whose illustration requires a $1000 \times 1000$ grid. To show the results in a manner more quantitative than is possible in Figs. 14 and 15, we consider slices through the 2D probability data taken along the $x$ axis, along the diagonal $x+y$, and along the "edge" of the data set, as shown in Fig. 16 .

The results of this process are illustrated in Fig. 17 for walks of $N=100$ steps. For the non-entangled case,

TABLE IV. Probability distributions of the 2D QW for $N=6$ steps, shown as $4096 P(x, y)$. On the left is the four-facecoin QW (equivalent to two unentangled coins) and on the right the AQW (equivalent to a single coin, or two maximally entangled coins). For the interpretation of these numbers we note that in the $1 \mathrm{D}$ QW with $N=6$ one obtains $64 P(x)=$ $[1,18,9,8,9,18,1]$.

\begin{tabular}{|c|ccccccc||ccccccc|}
\hline $\mathrm{x}$ & -6 & -4 & -2 & 0 & 2 & 4 & 6 & -6 & -4 & -2 & 0 & 2 & 4 & 6 \\
\hline 6 & 1 & 18 & 9 & 8 & 9 & 18 & 1 & 1 & 26 & 125 & 200 & 125 & 26 & 1 \\
4 & 18 & 324 & 162 & 144 & 162 & 324 & 18 & 26 & 68 & 50 & 208 & 50 & 68 & 26 \\
2 & 9 & 162 & 81 & 72 & 81 & 162 & 9 & 125 & 50 & 89 & 40 & 89 & 50 & 125 \\
0 & 8 & 144 & 72 & 64 & 72 & 144 & 8 & 200 & 208 & 40 & 64 & 40 & 208 & 200 \\
-2 & 9 & 162 & 81 & 72 & 81 & 162 & 9 & 125 & 50 & 89 & 40 & 89 & 50 & 125 \\
-4 & 18 & 324 & 162 & 144 & 162 & 324 & 18 & 26 & 68 & 50 & 208 & 50 & 68 & 26 \\
-6 & 1 & 18 & 9 & 8 & 9 & 18 & 1 & 1 & 26 & 125 & 200 & 125 & 26 & 1 \\
\hline
\end{tabular}

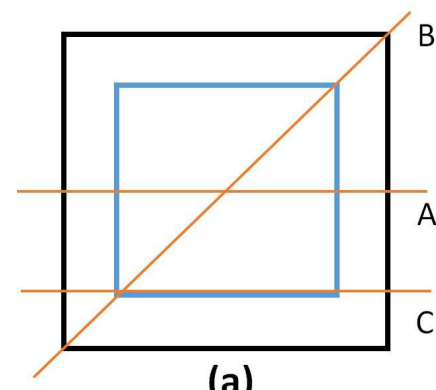

(a)

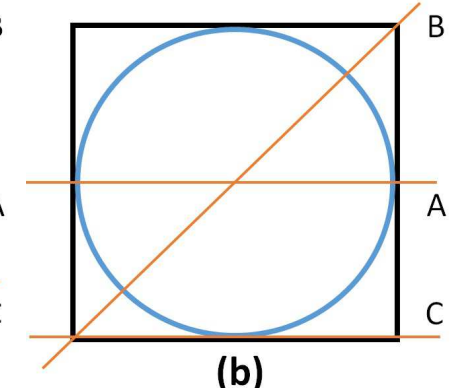

(b)
FIG. 16. Schematic illustration of 1D slices taken through the 2D probability distribution for the two 2D QWs considered, whose data are shown in Fig. 17. Slice A denotes the $x$ direction, $\mathrm{B}$ the diagonal, and $\mathrm{C}$ is the edge of data. In panel (a), where the blue square denotes the region of maximum probability, slice $\mathrm{C}$ is taken at $x=0.7 N$; in panel (b), where the maximum probability is found along the blue circle, slice $\mathrm{C}$ is taken at the true edge $(x=N)$.

panel A1 proves the numerical identity with the $1 \mathrm{D} \mathrm{QW}$, $P(x, 0)=P(x) P(0)$, which can be compared with Fig. 2 using the result for $P(0)$ with $N=100$. This being the case, panel $\mathrm{C} 1$ is very easy to interpret and for any parallel cut would have the same functional form with any prefactor from $P(x)$. Panel B1 can be expected to satisfy $P(x, x)=P^{2}(x)$, and therefore to have an envelope function of the form $(N / \sqrt{2}-x)^{-1}$ across the outer half of the distribution, a result we demonstrate below. By contrast, rather little is known about the distribution $P(x, y)$ for the AQW and its understanding will require applying the techniques of Sec. III, for small data sets, to the panels A2, B2, and C2 of Fig. 16. Qualitatively, in A2, the horizontal slice through the probability maxima, we observe an apparent envelope function with no oscillations and a divergence towards $x= \pm N$ with an unknown power. In B2 we observe a complex oscillatory pattern with significant probability extending beyond its peak value. In $\mathrm{C} 2$ we observe a remarkably classical-looking probability distribution at the edge of the system, where we remind the reader that the peak is the absolute maximum anywhere in the walk.

We wish to characterize the AQW by the power-law form of its probability envelope function around the peak values of the distribution. For this we analyze the 2D probability slices following Eq. (10), but because our largest system size in $2 \mathrm{D}$ is $N=1000$, the numerical results do not give particularly reliable curve fits. Although the divergence of $P(x)$ at $x=N / \sqrt{2}$ (at $x=N$ for slice A2) is not achieved with any accuracy for these values of $N$, we enforce this value in all cases other than slice $\mathrm{B} 2$ to reduce the arbitrariness in the fitting parameters. We show in Fig. 18 the probability on logarithmic axes as a function of $(b-x) / N$ for fixed values $b=0.707 N$ in cases $\mathrm{A} 1$ and $\mathrm{B} 1, b=N$ in case $\mathrm{A} 2$, and the fitted value $b=0.8 N$ in case B2. As expected, the probabilities for panels $\mathrm{A} 1$ and $\mathrm{B} 1$ have gradients close to $-1 / 2$ and -1 


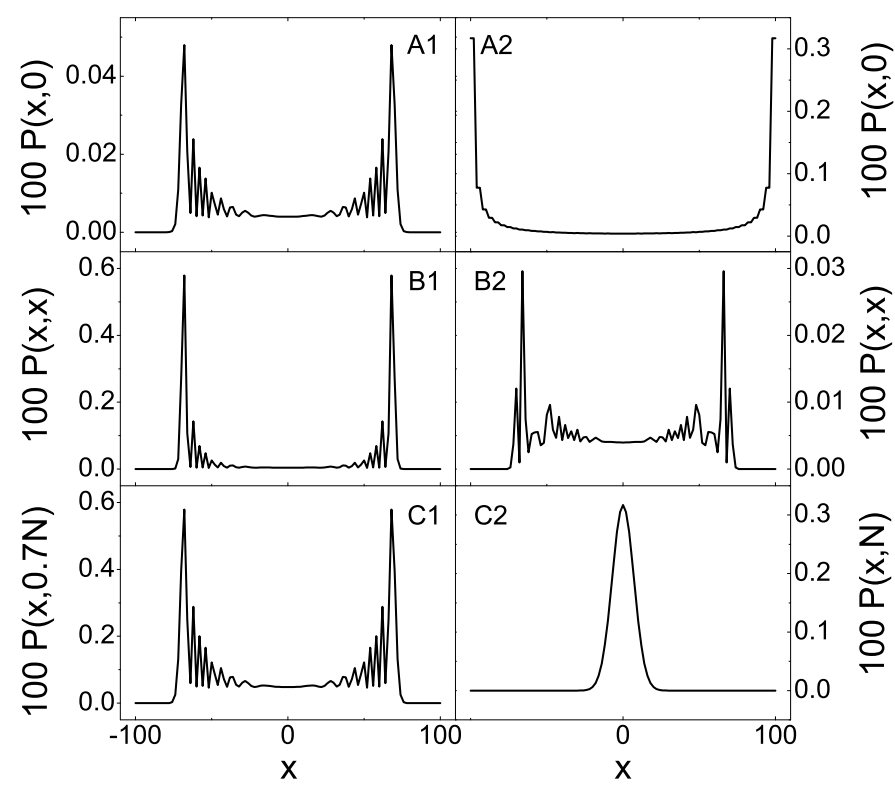

FIG. 17. Probability distributions on $1 \mathrm{D}$ slices through the 2D QW data (see Fig. 16) for walks of $N=100$. Panels A1, $\mathrm{B} 1$, and $\mathrm{C} 1$ are for the unentangled $\mathrm{QW}$ and $\mathrm{A} 2, \mathrm{~B} 2$, and $\mathrm{C} 2$ for the AQW.

$[c=1 / 2$ and $c=1$ in Eq. (10)], respectively, over the bulk of the range, and the accuracy to which the data fall on a straight line benchmarks the method for $N=1000$. For panel A2, the data are remarkably clean and give a clear gradient parameter $c=1$, indicating that the envelope function of the peak in the maximally entangled walk satisifes the form $P(x, 0)=P_{0}+a /(1-x / N)$ to high accuracy. For panel B2, a slice that does not include the main peaks but only the circular edge of local probability maxima, we find a result close to $c=1 / 2$, although here the envelope is poorly defined and the errors significant.

While our estimate of the functional form of the $x$ dependence of the AQW is somewhat approximate, the $N$-dependence of the probability distribution is beyond doubt. At every corresponding position, the probability falls by a factor of 4 for every doubling of $N$. This result is illustrated by the black $(N=500)$ and red $(N=1000)$ lines in Fig. 18. Thus we propose that the appropriate fit to the probability data on these four slices is given by the algebraic form

$$
y=\frac{a_{1}}{N^{2}}+\frac{a_{2}}{N^{d}}(b-x)^{-c},
$$

with $c+d=2$ and the values of $c$ as deduced from Fig. 18, We may conclude that the entangled 2D QW has algebraic scaling properties similar to the 1D-case. While its probability distribution shows no oscillations in the $x$ and $y$ directions, in the $x \pm y$ directions it oscillates in a manner not dissimilar to the 1D QW (panel B2 in Fig. 17). In Fig. 19, we show that the number of peaks in this slice is again proportional to the step number $N$, and with a constant of proportionality again of order $8.5 \%$ (Sec. IIIB),
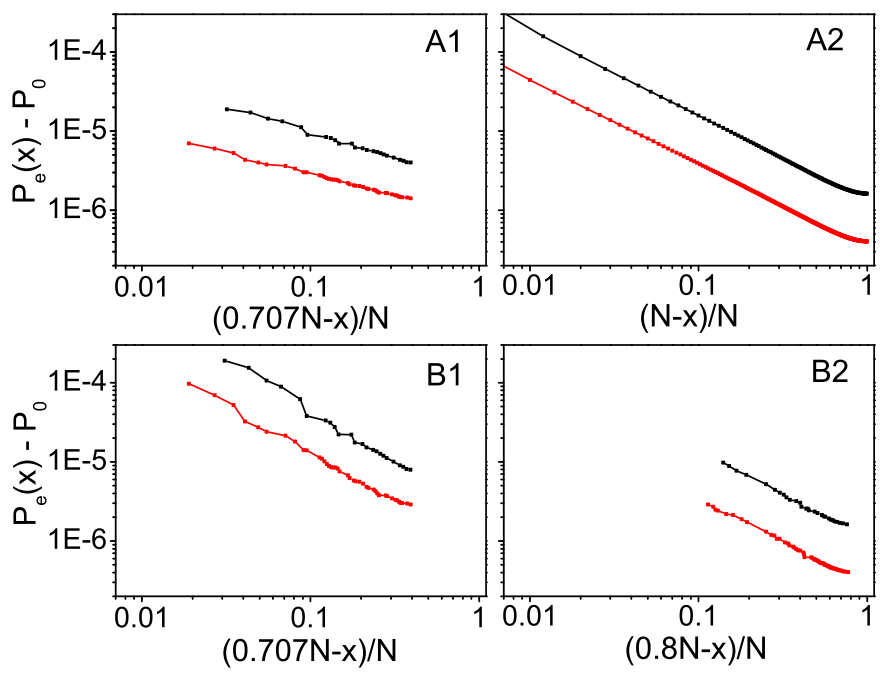

FIG. 18. (color online) Scaling properties of the envelope function of the $2 \mathrm{D}$ probability distribution for $N=500$ (black) and $N=1000$ (red) on the data slices shown in Fig. 17, panels A1, B1, A2, and B2.

although we point out that for $x \sim N / \sqrt{2}$, where the $1 \mathrm{D}$ QW shows its strongest peak, this distribution has its deepest valley.

\section{B. Edge of the Probability Distribution}

Next we consider the probability distribution at the edge of the walk for the AQW, shown in panel C2 of Fig. 17. As remarked above, this QW has the highly anomalous feature that its maximum probability occurs when the walker takes the maximum number of steps in the same direction along one of the two axes, but returns to the center of the other axis. Further, a visual inspection of the 1D slice through this maximum suggests that the distribution on this edge may be a Gaussian, or a related function similar to the 1D classical random walk.

To investigate whether a quantum walk can lead to a classical results, we employ the decomposition of the unitary evolution matrix into the matrices $P$ and $Q$ introduced in Sec. 【IB, For the $2 \mathrm{D}$ QW we require matrices $P_{x}$ for steps to the left, $Q_{x}$ for right, $P_{y}$ for up, and $Q_{y}$ for down. Exploiting the AQW equivalence of the maximally entangled two-coin QW, we use the same coin alternately for the $x$ and $y$ directions. Because our aim is to understand the probability distribution on the right-hand edge of the system (equivalent to Fig. 16)(b), slice C), every $x$-direction operation for the walker must be a $Q_{x}$ matrix and not $P_{x}$. A complete cycle of the walk may then be either $R=P_{y} Q_{x}$ or $S=Q_{y} Q_{x}$, where

$$
\begin{aligned}
& R=P_{y} Q_{x}=\frac{1}{2}\left(\begin{array}{ll}
1 & 1 \\
0 & 0
\end{array}\right)\left(\begin{array}{cc}
0 & 0 \\
1 & -1
\end{array}\right)=\frac{1}{2}\left(\begin{array}{cc}
1 & -1 \\
0 & 0
\end{array}\right), \\
& S=Q_{y} Q_{x}=\frac{1}{2}\left(\begin{array}{cc}
0 & 0 \\
-1 & 1
\end{array}\right)\left(\begin{array}{cc}
0 & 0 \\
1 & -1
\end{array}\right)=\frac{1}{2}\left(\begin{array}{cc}
0 & 0 \\
1 & -1
\end{array}\right),
\end{aligned}
$$




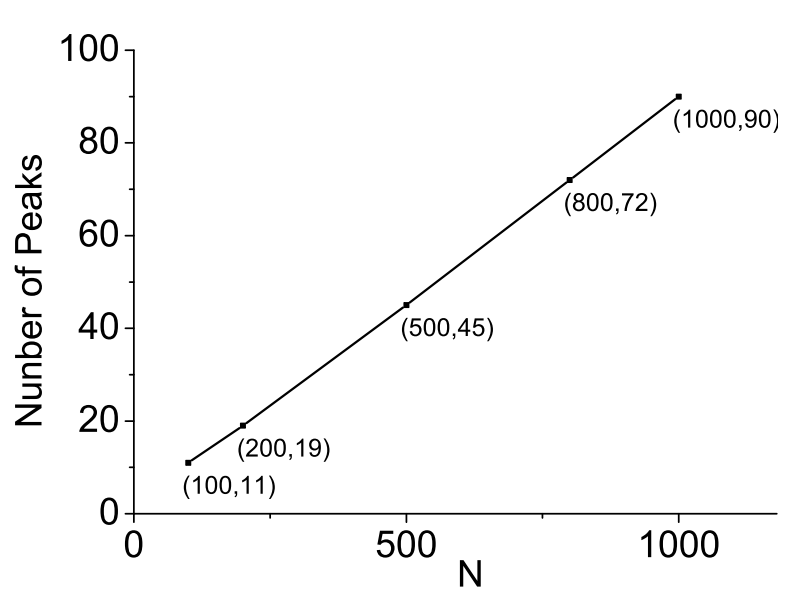

FIG. 19. Number of peaks in the probability distribution of the AQW (maximally entangled two-coin QW) in the direction $x+y$, shown in panel B2 of Fig. 17 as a function of step number $N$.

and thus

$$
\begin{aligned}
& R R=\frac{1}{4}\left(\begin{array}{cc}
1 & -1 \\
0 & 0
\end{array}\right)\left(\begin{array}{cc}
1 & -1 \\
0 & 0
\end{array}\right)=\frac{1}{4}\left(\begin{array}{cc}
1 & -1 \\
0 & 0
\end{array}\right)=\frac{1}{2} R, \\
& R S=\frac{1}{4}\left(\begin{array}{cc}
1 & -1 \\
0 & 0
\end{array}\right)\left(\begin{array}{cc}
0 & 0 \\
-1 & 1
\end{array}\right)=\frac{1}{4}\left(\begin{array}{cc}
1 & -1 \\
0 & 0
\end{array}\right)=\frac{1}{2} R, \\
& S R=\frac{1}{4}\left(\begin{array}{cc}
0 & 0 \\
-1 & 1
\end{array}\right)\left(\begin{array}{cc}
1 & -1 \\
0 & 0
\end{array}\right)=\frac{1}{4}\left(\begin{array}{cc}
0 & 0 \\
-1 & 1
\end{array}\right)=\frac{1}{2} S, \\
& S S=\frac{1}{4}\left(\begin{array}{cc}
0 & 0 \\
-1 & 1
\end{array}\right)\left(\begin{array}{cc}
0 & 0 \\
-1 & 1
\end{array}\right)=\frac{1}{4}\left(\begin{array}{cc}
0 & 0 \\
-1 & 1
\end{array}\right)=\frac{1}{2} S,
\end{aligned}
$$

i.e. only the left-most operator in the string of steps is important.

With this result it is possible to calculate the entire edge distribution analytically for any value of $N$. We illustrate the process for a walk of $N=4$ steps. The paths arriving at position $(N, 0)$ are $R R S S, R S S R, R S R S$, $S S R R, S R S R$, and $S R R S$, which are divided between the coin states $|\uparrow\rangle$ and $|\downarrow\rangle$ according to

$$
\begin{aligned}
& U_{|\uparrow\rangle}^{(N, 0)}=R R S S+R S S R+R S R S=\frac{1}{16}\left(\begin{array}{cc}
3 & -3 \\
0 & 0
\end{array}\right), \\
& U_{|\downarrow\rangle}^{(N, 0)}=S S R R+S R S R+S R R S=\frac{1}{16}\left(\begin{array}{cc}
3 & -3 \\
0 & 0
\end{array}\right),
\end{aligned}
$$

whence

$$
P_{|\uparrow\rangle}^{(N, 0)}=\left|\frac{3-3 i}{16 \sqrt{2}}\right|=\frac{9}{256}, \quad P_{|\downarrow\rangle}^{(N, 0)}=\left|\frac{3-3 i}{16 \sqrt{2}}\right|=\frac{9}{256},
$$

and finally

$$
P^{(N, 0)}=P_{|\uparrow\rangle}^{(N, 0)}+P_{|\downarrow\rangle}^{(N, 0)}=\frac{18}{256} .
$$

Paths arriving at position $(N, 2)$ are $R R R S, R R S R$,
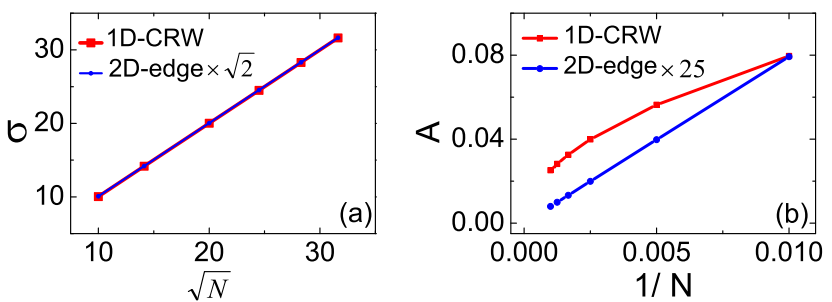

FIG. 20. Comparison between the AQW edge probability distribution and a binomial (classical random walk) distribution for values up to $N=1000$ steps, where the binomial is very accurately Gaussian. (a) Standard deviation $\sigma$. (b) Prefactor $A$.

$R S R R$, and $S R R R$, yielding

$$
\begin{aligned}
& U_{|\uparrow\rangle}^{(N, 2)}=R R R S+R R S R+R S R R=\frac{1}{16}\left(\begin{array}{cc}
3 & -3 \\
0 & 0
\end{array}\right), \\
& U_{|\downarrow\rangle}^{(N, 2)}=S R R R=\frac{1}{16}\left(\begin{array}{cc}
0 & 0 \\
-1 & 1
\end{array}\right), \\
& P_{|\uparrow\rangle}^{(N, 2)}=\left|\frac{3-3 i}{16 \sqrt{2}}\right|=\frac{9}{256}, \quad P_{|\downarrow\rangle}^{(N, 2)}=\left|\frac{-1+i}{16 \sqrt{2}}\right|=\frac{1}{256},
\end{aligned}
$$

and thus

$$
P^{(N, 2)}=P_{|\uparrow\rangle}^{(N, 2)}+P_{|\downarrow\rangle}^{(N, 2)}=\frac{10}{256} .
$$

The only path arriving at position $(N, 4)$ is $R R R R$, leading to

$$
\begin{aligned}
& U_{|\uparrow\rangle}^{(N, 4)}=R R R R=\frac{1}{16}\left(\begin{array}{cc}
1 & -1 \\
0 & 0
\end{array}\right), \\
& P^{(N, 4)}=P_{|\uparrow\rangle}^{(N, 4)}=\left|\frac{1-i}{16 \sqrt{2}}\right|=\frac{1}{256} .
\end{aligned}
$$

This analytical solution demonstrates that the quantum mechanical interference leading to the probability on the edge of the system is constructive everywhere, with none of the paths interfering destructively. Regions at the center of the edge simply have the most paths, and this is the origin of what we can call the "semi-classical" result that the probability is maximal at the center of the edge, leading to a distribution similar in appearance to the binomial distribution of the classical random walk. In fact the degree of destructive interference everywhere else in the 2D AQW is such that these maxima on the edges are the global maxima, meaning that the walker is effectively pushed to the maximal number of steps in order that it does not "destroy itself" by interference in the maximally entangled two-coin walk.

A quantitative examination of the coefficients of the AQW edge reveals that, despite being peaked at the center, they are not the same as the 1D classical random walk, as shown in Table $\mathrm{V}$ The probability distribution at position $x$ on the edge of an $N$-step AQW can in fact 
be expressed exactly as

$$
\begin{aligned}
P_{N}(N, x) & =\frac{1}{4}\left[P_{N-1}^{2}(x-1)+P_{N-1}^{2}(x+1)\right] \\
& =\frac{1}{4^{N}}\left(\left[C_{N-1}^{\frac{N-x-1}{2}}\right]^{2}+\left[C_{N-1}^{\frac{N-x+1}{2}}\right]^{2}\right),
\end{aligned}
$$

where $C_{n}^{i}$ is a binomial coefficient (Sec. IIA). This remarkable result, which we term a "pseudobinomial" distribution, encodes the emergence of the physics of classical probabilities in the highly entangled QW. The binomial coefficients appearing in the penultimate step before measurement (Table V]) arise as a consequence of the complete constructive interference of paths, as illustrated in Eqs. (22) to (24) and discussed in the preceding paragraph.

To demonstrate the form of the AQW edge probability at large $N$, we fit both binomial [Eq. (1)] and pseudobinomial distributions to the Gaussian, as specified in Eq. (9) and again with $P_{0}=0=b$, to compare their forms and to extract their standard deviations $\sigma$. As shown in Fig. 20(a), while the binomial distribution gives the result $\sigma=\sqrt{N}$, the standard deviation of the AQW edge probability is $\sigma=\sqrt{N / 2}$, i.e. the distribution is narrower and the diffusion, or spreading, rate of the walk slower (more localized) by a factor of $1 / \sqrt{2}$. Concerning the normalization prefactor $A$ [Fig. 20(b)], the binomial approaches the Gaussian result $A=1 / \sqrt{2 \pi N}$, but the AQW edge distribution does not, following instead a dependence $A \propto 1 / N$. While the probability of the classical random walk of course sums to unity, determining $A$ for a true Gaussian, the probability distribution at the edge of the AQW is not normalized due to the weight in the interior of the walk, and hence the edge probability is found

TABLE V. Probabilility tables for the first 7 steps of the binomial distribution (top), omitting a prefactor of $1 / 2^{N}$ at each level $N$ of the table, and for the pseudobinomial distribution (bottom) achieved on the edges of the 2D AQW, omitting a prefactor of $1 / 4^{N}$.

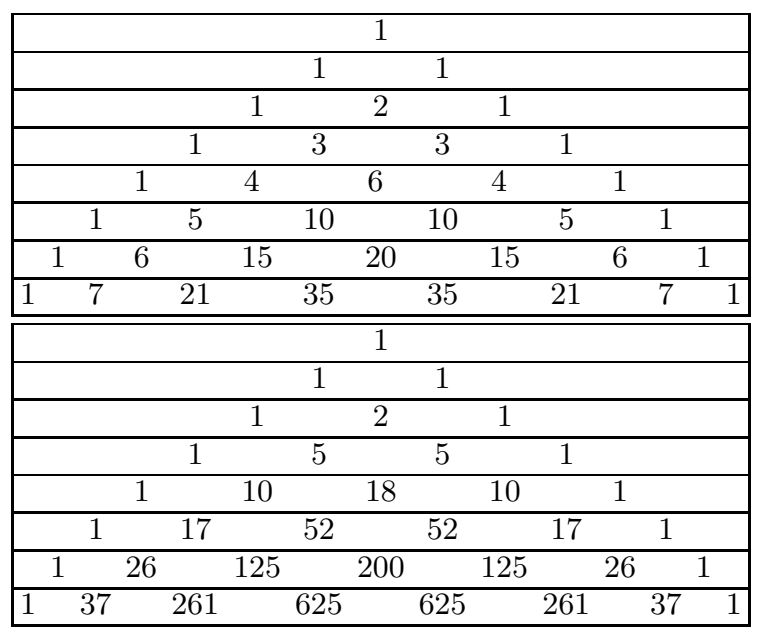
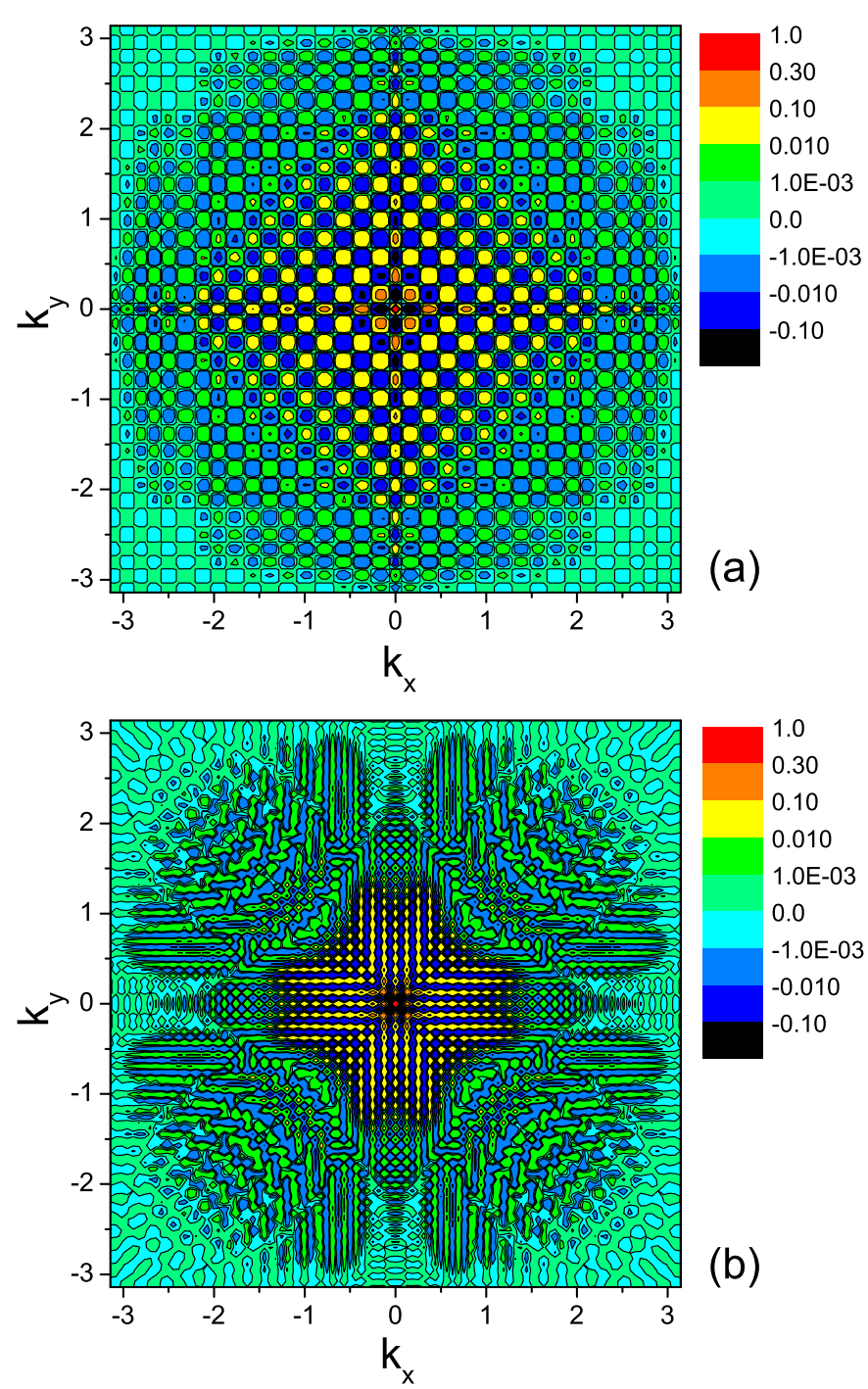

FIG. 21. 2D Fourier transform $F\left(k_{x}, k_{y}\right)$ of the probability distributions $P(x, y)$ (Fig. ??) for (a) the four-face-coin or unentangled 2D QW and (b) the 2D AQW or maximally entangled two-coin QW, both for $N=100$.

to decay more rapidly than a true Gaussian. The emergence of such a pseudobinomial distribution in a $\mathrm{QW}$ is yet another example of the rich physics contained in a deceptively simple quantum evolution algorithm.

\section{Fourier Transformation of 2D QWs}

For further insight into the structure of the AQW probability distribution, we compute the Fourier transform $F\left(k_{x}, k_{y}\right)$ of $P(x, y)$ for both the 2D QWs we consider. Figure 21(a) shows the 2D Fourier transform of the unentangled (four-face-coin) QW, whose product structure is again clearly visible. It was shown in Sec. III that the 1D QW has spatial frequency information at all scales up to the inverse step size $(k=\pi)$ and this is clear in the 


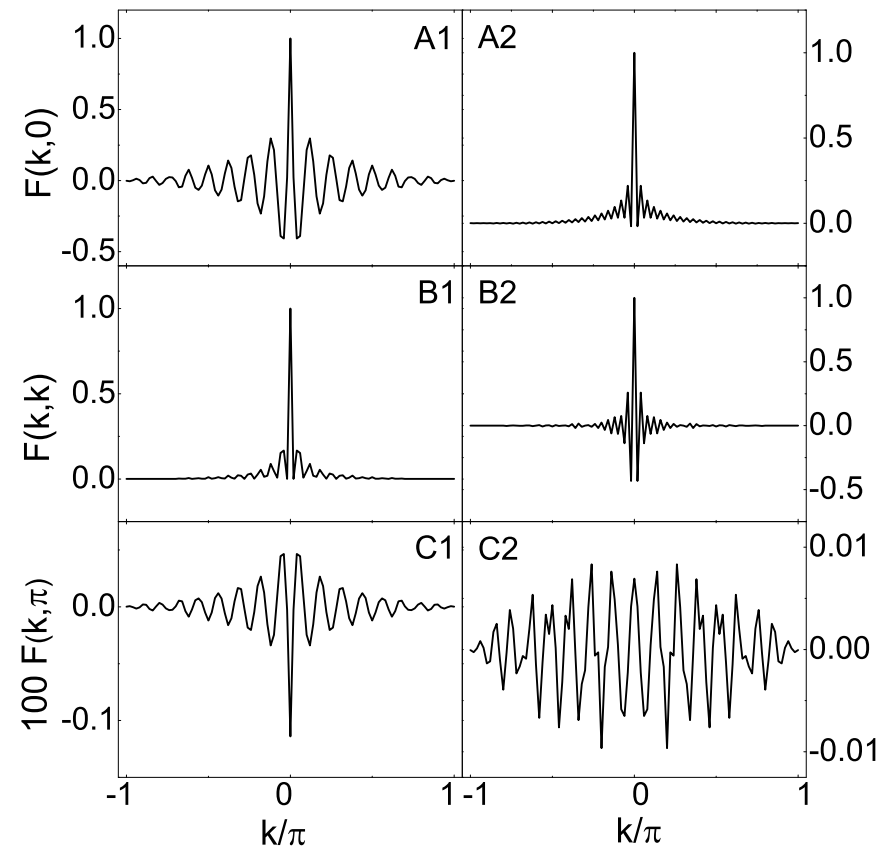

FIG. 22. Cross-sections of the 2D Fourier transform $F\left(k_{x}, k_{y}\right)$ of the probability distributions [Fig. 17] for the two 2D QWs of Sec. IV, calculated with $N=100$ and shown for the slices studied in Sec. VA (Fig. 16).

finite components of $F\left(k_{x}, k_{y}\right)$ up to the edge of Fourier space. The AQW, shown in Fig. 21(b), is again quite different, showing both an intrinsically $2 \mathrm{D}$ character and an apparent concentration of weight closer to the center of Fourier space.

To analyze these distributions in a quantitative manner, we present the data in the form of $1 \mathrm{D}$ slices. Figure 22 is completely analogous to Fig. 17, with the unentangled QW in the left panels, the entangled one on the right, A denoting a horizontal slice through the center of the Fourier distribution, $\mathrm{B}$ a diagonal slice, and $\mathrm{C}$ the edge. We illustrate the qualitative features of the data using QWs of $N=100$ steps, but for our numerical analysis of the properties of the Fourier transforms we use values of $N$ up to 1000 .

In panel A1 of Fig. 22, which shows $F\left(k_{x}, 0\right)$ for the unentangled $\mathrm{QW}$, we observe the factorized form $F\left(k_{x}\right) F(0)=F\left(k_{x}\right)$ expected from Sec. VA and shown in Fig. 3(b). Panel B1 shows $F\left(k_{x}, k_{x}\right)=F^{2}\left(k_{x}\right)$, with only positive components and a correspondingly steeper decay of the envelope. Panel $\mathrm{C} 1$, showing $F\left(\pi, k_{y}\right)$, is identical to A1 up to a multiplicative prefactor, which is small and happens to be negative at $k_{x}=\pi$. Turning to the AQW, where the probability distribution cannot be factorized, the slice $F\left(k_{x}, 0\right)$ in panel A2 is not dissimilar to panel B1, in that all of the Fourier components are positive and they decay significantly more rapidly than those of the 1D QW. However, their oscillatory form shows a very precise odd/even modulation, which is not evident in the $1 \mathrm{D} \mathrm{QW}$. The diagonal slice $F\left(k_{x}, k_{x}\right)$ is shown in panel B2 and confirms both the odd/even modulation and the concentration of Fourier amplitude near the center of the system. Finally, panel $\mathrm{C} 2$ has no readily discernible structure, reflecting the fact that the AQW edge is non-oscillatory and thus dramatically different from all the other QW distribution slices (Sec. VB).

Following Sec. IIID, we investigate two properties of the Fourier transform slices, namely their envelope and their oscillations. To characterize the decay of the envelope function, we follow the procedure shown in Fig. 13 and use logarithmic axes. As shown in Fig. 223(a), and as expected from Sec. IIID, the envelope functions for panels A1 and B1 yield algebraic decay exponents close to $c=1 / 2$ and $c=1$, respectively, for the Fourier components around $k=0$; the accuracy with which the data adhere to a straight line again benchmarks the accuracy one may expect from data up to $N=1000$. The data from slice A2 form by far the best-quality set in Fig. 223(a) and the algebraic decay exponent is unequivocally $c=1 / 2$, meaning that the spatial periodicity content of the AQW is qualitatively similar to that of the unentangled QW at low frequencies. We take this opportunity to remind the reader that there is a priori no direct connection between the real- and reciprocal-space exponents of the envelope functions (respectively around the peak of $P(x)$ and around $k=0$ ) and the two QWs present an example pair with different exponents in real space, describing the peak shape, but the same exponent in reciprocal space, describing the frequency content. The data from slice B2 exhibit the lowest-quality envelope in the figure, but still show a strong qualititive similarity to slice B1, with $c=1$. Again this highlights the complexity of the spatial frequency content of the AQW in its different directions and indicates that the entanglement inherent in the AQW also entangles the spatial information of the different lattice directions.

For completeness, we show in Fig. 23(b) the Fourier components of the four slices at high $k$, where once again slices $\mathrm{A} 1$ and $\mathrm{B} 1$, with respective gradients $c^{\prime}=1$ and $c^{\prime}=2$ benchmark the accuracy of the approach. Again we observe that slices A2 and B2 for the AQW are qualitatively similar, with the caveat that the data for slice A2 show evidence of a distinctively different intermediate regime. We stress that the difference in amplitude of the Fourier components between the unentangled QW and the AQW, which reaches two orders of magnitude over the large- $k$ half of the range for slices A1 and A2, is the most meaningful measure of strong quantitative differences between the two QWs.

Concerning the oscillations visible in the Fourier transforms, in the unentangled case it is known from Sec. III that the 2D QW contains spatial frequency information on all length scales and that beating phenomena become visible both in real space around the maximum frequency and as a result in Fourier space near $k= \pm \pi$. For the AQW, our data (Figs. 22] and 231) show that the high-frequency spatial components are very significantly 

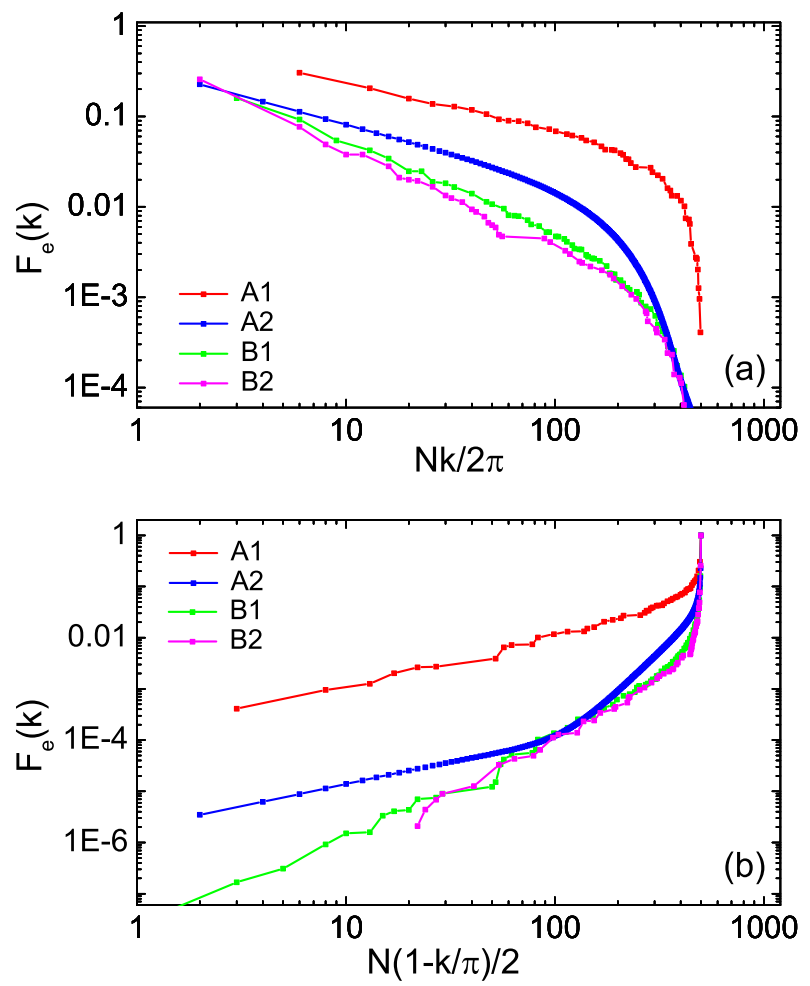

FIG. 23. Fourier transform of the 2D probability distributions for slices A1, B1, A2, and B2 shown in Fig. 17 (a) as a function of $k$, showing algebraic decay of the small- $k$ components, and (b) as a function of $1-k / \pi$, showing different power-law dependences at large $k$.

weaker, meaning that lattice-scale oscillations are of little relevance, and this suggests that beating (which we are unable to find up to $N=1000$ ) is unlikely to be present. We believe that the extra strength of even harmonics in the AQW results from the even step number.

We close our analysis of the Fourier-space information contained in a QW by summarizing a complementary perspective, named the "dispersion relation" approach in Ref. [31]. In Sec. IIC we presented the exact solution of the 1D QW by considering its nature in Fourier space, finding that the Hadamard operator (3) gave rise to a dispersion relation for a quantity $\omega_{k}=\sin ^{-1}[\sin k / \sqrt{2}]$, specified in terms of $k$. Although $\omega_{k}$ is largely a simplifying notation, as there is no concept of a separate "time" (step number) and space in a QW, it does result in a compact description in higher dimensions and it has the added advantage of reflecting different degrees of coin entanglement in a transparent way. When the AQW in 2D is transformed into Fourier space [31], one may express the operator as

$G_{4}=\frac{1}{2}\left(\begin{array}{cccc}-e^{i k_{1}} e^{i k_{2}} & e^{i k_{1}} e^{i k_{2}} & e^{i k_{1}} e^{i k_{2}} & e^{i k_{1}} e^{i k_{2}} \\ e^{i k_{1}} e^{-i k_{2}} & -e^{i k_{1}} e^{-i k_{2}} & e^{i k_{1}} e^{-i k_{2}} & e^{i k_{1}} e^{-i k_{2}} \\ e^{-i k_{1}} e^{i k_{2}} & e^{-i k_{1}} e^{i k_{2}} & -e^{-i k_{1}} e^{i k_{2}} & e^{-i k_{1}} e^{i k_{2}} \\ e^{-i k_{1}} e^{-i k_{2}} & e^{-i k_{1}} e^{-i k_{2}} & e^{-i k_{1}} e^{-i k_{2}} & -e^{-i k_{1}} e^{-i k_{2}}\end{array}\right)$ and deduce the eigenvalues

$$
\begin{aligned}
\lambda_{k}^{1 \pm} & = \pm 1 \\
\lambda_{k}^{2 \pm} & =-\frac{1}{2}\left[\cos \left(k_{1}+k_{2}\right)+\cos \left(k_{1}-k_{2}\right)\right. \\
& \left. \pm \sqrt{\left(\cos \left(k_{1}+k_{2}\right)+\cos \left(k_{1}-k_{2}\right)\right)^{2}-4}\right] .
\end{aligned}
$$

Because $\lambda_{k}=e^{i \omega_{k}}$, the dispersion relations for the four eigenmodes of the AQW take the form

$$
\begin{aligned}
& \omega_{k}^{1+}=0, \quad \omega_{k}^{1-}=\pi, \\
& \omega_{k}^{2 \pm}=\pi \mp \frac{1}{2}\left(\cos k_{1}+\cos k_{2}\right),
\end{aligned}
$$

allowing a considerable simplification of the process [Eq. (7)] of generating the probability distribution in this case.

\section{SUMMARY}

Quantum walks produce probability distributions entirely different from the well-known classical "drunkard's walk." In most cases, the distribution is controlled completely by predominantly destructive interference between the paths returning to the center, with the result that the positions of maximal probability are pushed out towards the edges of the walk. This results in the "linear diffusion" property of a quantum walker, which has made the QW evolution algorithm so valuable in studies of quantum computing.

However, as quantum computing approaches largescale implementation, it is necessary to understand the nature of quantum algorithms at large scales. By this is meant an exact account of their properties, information content, limiting behavior, and scaling characteristics at long evolution times. Although analytical studies of different quantum walks in one and two dimensions have revealed certain characteristics of their evolution and interference, in particular their dependence on the initial state and the entanglement of the quantum coins generating them, to date there has been rather little consideration of the situation at large step number $N$.

By calculating the probability distributions for oneand two-dimensional quantum walks up to $N=1000000$ in the former case and $N=1000$ in the latter, we have revealed a number of properties and scaling characteristics. In one dimension, we verify that the probability approaches peaks at $x / N= \pm 1 / \sqrt{2}$ at large $N$, which is the transition region between destructive interference and vanishing probability for all steps to be made in the same direction. The normalized support converges to the region $[-1 / \sqrt{2}, 1 / \sqrt{2}]$ and the envelope of the distribution peaks has a square-root decay, i.e. an algebraic form. Within this envelope, the probability shows systematic oscillations on all length scales, with the number of probability peaks always the same fraction of $N$. The frequency of these oscillations increases from the inverse system size to the inverse step length as a function of 
distance from the center of the walk. The different frequencies show complex beating phenomena in the regime where the oscillations are most rapid. These properties are revealed in a complementary fashion by taking the spatial Fourier transform of the distribution. All of these features are universal for walks of all $N$ values, giving them very strong similarities, but not the property of self-similarity (there are no fractal structures in the simple walks studied here).

In two dimensions there is not one quantum walk, or even one algorithm, but a spectrum of protocols capable of generating quantum evolution in a plane of phase space. We study two examples that in fact represent the limiting cases of the entanglement between two orthogonal directions: unentangled 2D QW and the maximally entangled alternate quantum walk(AQW).

In our numerical studies of these two limits, the understanding developed in one dimension gives a complete account of the unentangled quantum walk, whose twodimensional probability distribution factorizes exactly into two one-dimensional functions. By contrast, the maximally entangled case exhibits strong correlations between the two orthogonal directions, damping of the oscillatory behavior, and the extraordinary feature that the maximum of the probability distribution is pushed all the way to the system edge by the dominance of destructive interference. We provide an analytical description of the edge distribution, showing that all paths arriving at the system edges interfere constructively and proving that its functional form is a type of pseudo-binomial, which is semi-classical in the sense of approaching a Gaussian dependence on the spatial coordinate at large $N$.

As two-dimensional quantum walks become an experimental science, our analytical and numerical studies demonstrate that even the simplest algorithms for quantum evolution contain a rich variety of physical phenomena and potential for technological application.

\section{ACKNOWLEDGMENTS}

The authors gratefully acknowledge helpful discussions with Professor B.Normand. Work at Renmin University was supported by the National Natural Science Foundation of China (NSFC) under Grant No. 11174365 and by the National Basic Research Program of China (NBRPC) under Grant No. 2012CB921704. PX was supported by the NSFC under Grant Nos. 11174052 and 11474049, by the NBRPC under Grant No. 2011CB921203, by the Open Fund from the State Key Laboratory of Precision Spectroscopy of East China Normal University, and by the CAST Innovation Fund.
[1] J. Kempe, Contemporary Physics 44, 307 (2003).

[2] Y. Aharonov, L. Davidovich, and N. Zagury, Phys. Rev. A 48, 1687 (1993).

[3] N. Shenvi, J. Kempe, and K. B. Whaley, Phys. Rev. A 67, 052307 (2003).

[4] A. Ambainis, Int. J. Quantum Inf. 1, 507 (2003).

[5] V. Kendon, Phil. Trans. R. Soc. A 364, 3407 (2006).

[6] A. M. Childs, R. Cleve, E. Deotto, E. Farhi, S. Gutmann, and D. A. Spielman, in Proceedings of the thirty-fifth Annual ACM Symposium on Theory of Computing, STOC '03 (ACM, New York, NY, USA, 2003) pp. 59-68.

[7] J. Kempe, in Proceedings of the 7th International Workshop on Randomization and Approximation Techniques in Computer Science (RANDOM03) (2003) pp. 354-369.

[8] A. Ambainis, J. Kempe, and A. Rivosh, in Proceedings of 16th ACM-SIAM SODA (2005) pp. 1099-1108.

[9] A. Ambainis, E. Bach, A. Nayak, A. Vishwanath, and J. Watrous, in Proceedings of the thirty-third Annual ACM Symposium on Theory of Computing, STOC '01 (ACM, New York, NY, USA, 2001) pp. 37-49.

[10] A. M. Childs, E. Farhi, and S. Gutmann, Quantum Inf. Process. 1, 35 (2002).

[11] B. Tregenna, W. Flanagan, R. Maile, and V. Kendon, New J. Phys 5, 83 (2003).

[12] P. Xue and B. C. Sanders, Phys. Rev. A 85, 022307 (2012).

[13] T. D. Mackay, S. D. Bartlett, L. T. Stephenson, and B. C. Sanders, J. of Phys. A: Math. Gen. 35, 2745 (2002).

[14] P. Xue, H. Qin, B. Tang, and B. C. Sanders, New J. Phys. 16, 053009 (2014).
[15] C. Di Franco, M. McGettrick, T. Machida, and T. Busch, Phys. Rev. A 84, 042337 (2011).

[16] C. Di Franco, M. McGettrick, and T. Busch, Phys. Rev. Lett. 106, 080502 (2011).

[17] B. Do, M. L. Stohler, S. Balasubramanian, D. S. Elliott, C. Eash, E. Fischbach, M. A. Fischbach, A. Mills, and B. Zwickl, J. Opt. Soc. Am. B 22, 499 (2005).

[18] A. Schreiber, K. N. Cassemiro, V. Potoček, A. Gábris, P. J. Mosley, E. Andersson, I. Jex, and C. Silberhorn, Phys. Rev. Lett. 104, 050502 (2010).

[19] P. Zhang, X.-F. Ren, X.-B. Zou, B.-H. Liu, Y.-F. Huang, and G.-C. Guo, Phys. Rev. A 75, 052310 (2007).

[20] P. Zhang, B.-H. Liu, R.-F. Liu, H.-R. Li, F.-L. Li, and G.-C. Guo, Phys. Rev. A 81, 052322 (2010).

[21] M. Karski, L. Förster, J.-M. Choi, A. Steffen, W. Alt, D. Meschede, and A. Widera, Science 325, 174 (2009).

[22] H. Schmitz, R. Matjeschk, C. Schneider, J. Glueckert, M. Enderlein, T. Huber, and T. Schaetz, Phys. Rev. Lett. 103, 090504 (2009).

[23] F. Zähringer, G. Kirchmair, R. Gerritsma, E. Solano, R. Blatt, and C. F. Roos, Phys. Rev. Lett. 104, 100503 (2010).

[24] C. A. Ryan, M. Laforest, J. C. Boileau, and R. Laflamme, Phys. Rev. A 72, 062317 (2005).

[25] H. B. Perets, Y. Lahini, F. Pozzi, M. Sorel, R. Morandotti, and Y. Silberberg, Phys. Rev. Lett. 100, 170506 (2008).

[26] A. Schreiber, A. Gábris, P. P. Rohde, K. Laiho, M. Stefanak, V. Potoček, C. Hamilton, I. Jex, and C. Silberhorn, Science 336, 55 (2012).

[27] N. Konno, J. Math. Soc. Japan 57, 1179 (2005). 
[28] G. Grimmett, S. Janson, and P. F. Scudo, Phys. Rev. E 69, 026119 (2004).

[29] A. C. Oliveira, R. Portugal, and R. Donangelo, Phys. Rev. A 74, 012312 (2006).
[30] N. Inui, Y. Konishi, and N. Konno, Phys. Rev. A 69, 052323 (2004).

[31] E. Roldán, C. Di Franco, F. Silva, and G. J. de Valcárcel, Phys. Rev. A 87, 022336 (2013). 